\title{
Radiation Induced Darkening of the Optical Elements In the Startracker Camera
}

\author{
Richard H. White and G. Richard Wirtenson \\ University of California \\ Lawrence Livermore National Laboratory \\ Livermore, CA 94550
}

March 1993

This is an informal report intended primarily for internal or limited external distribution. The opinions and conclusions stated are those of the author and may or may not be those of the Laboratory.

- Work performed under the auspices of the U.S. Department of Encrgy by the Lawrence Livermore National Laboratory under Contract W-7405-Eng-48. 
This document was prepared as an scocount of wodk eponsored by an agency of the United States Covernment. Nelther the United States Government nor the Univerilty of Cilifornia nor any of their employeen, makes any warranty, expres or implled, or acoumes any legal liability or reoponaibility for the acourngy,

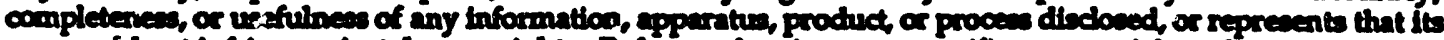
use would not infinge pstvately own rights. Reference heretn to any spedfic commendil products, proces, or service by trade name, trademarte, manufincture, or otherwite, does not neceavarlly constitute or imply its endorsement, recommendition, or favoring by the United States Covernment or the Univerilty of Calliornita. The views and opinions of authors expresed herela do not necesenrily etate or reflect those of the United States Covernment or the Univeratly of Callfornia, and shall not be used for advertiaing or product endorsement purpoces.

This report has been reproduced directly from the best avallable copy.

Available to DOE and DOE contractors from the Office of Salentific and Technical Information

P.O. Box 62, Onk Ridge, IN 37831

Prices available from (615) 576-8401, FTS 626-8401

Avallable to the public from the

National Technical Information Service

US. Department of Commerce 5285 Pat Royal Rd. Springfield, VA 22161 


\title{
Radiation Induced Darkening of the Optical Elements In the Startracker Camera
}

\author{
Richard H. White and G. Richard Wirtenson \\ University of California \\ Lawrence Livermore National Laboratory \\ P.O. Box 808, L-298 \\ Livermore, CA 94550
}

\begin{abstract}
Optical glass flats that closely simulate the elements used in the Startracker lens designs were exposed to doses of ionizing radiation ranging from 0.44 to $1300 \mathrm{krad}$. Photometer traces determined the transmittance of the samples as a function of both wavelength and dose for wavelengths in the range 300 to $1200 \mathrm{~nm}$. Cerium stabilized glasses used in the radiation stabilized Startracker system showed only a small amount of darkening for doses up to and exceeding $1 \mathrm{Mrad}$. Glasses used in the unstabilized Startracker design showed significant darkening to visible and ultra-violet spectra for doses as low as $\mathbf{5} \mathrm{krad}$. Plots of transmittance versus wavelength for various doses are given for each of the Startracker optical elements. Radiation induced absorption parameters that determine the radiation induced absorption coefficient are tabulated and plotted versus wavelength.
\end{abstract}

\section{INTRODUCTION}

The Startracker spacecraft navigational system includes optical glass elements that may be affected by the ionizing radiation present in the natural space environment. Ordinary optical glasses darken when exposed to ionizing radiation. This darkening decreases the sensitivity of optical sensors. Further, it decreases the apparent brightness of sources relied upon for navigation and so it may cause navigational errors due to false identification of these sources. It may also shift the apparent color of sources toward the red since darkening is generally greater for shorter wavelengths.

Radiation induced absorption coefficients have not previously been measured and reported for most of the glasses used in the Startracker system. Therefore, we exposed glass samples, of the same 
type as occur in the Startracker system, to specific doses of radiation and then measured the effect of the radiation on the transmittance of these glasses. In most cases the sample thickness equaled the axial thickness of, the corresponding Surtracker element.

The effect of ionizing radiation on the optical transmittance of irradiated materials, to a large extent, depends only on the dose delivered, regardless of the type of radiation exposure. (Wirtenson/White 1992) Therefore, one can choose a convenient source for the radiation without concern that it does not accurately model the radiation of the natural spacecraft environment. The Lawrence Livermore National Laboratory's $\mathrm{Co}^{60}$ source provided the ionizing radiation in these experiments. The primary source is 1.17 and 1.33 Mev $\gamma$ radiation.

In this report, we display the transmittances as a function of wavelength for the various Startracker optical elements and for radiation exposures ranging from 0.44 to $1300 \mathrm{krad}$.

\section{RADIATION INDUCED ABSORPTION}

Optical materials may darken when exposed to ionizing radiation. This darkening is attributed to electrons (or holes) that are trapped in inherent defect sites and also to the creation of new defect sites by the ionizing radiation. The darkening has been modeled as follows:

$$
T(\lambda, D, x)=T_{0} e^{-\alpha_{D} x}
$$

where $\mathrm{T}$ is the transmittance at wavelength $\lambda$ through an optical path length $\mathrm{x}$ of material that has received an accumulated radiation dose $D, T_{0}$ is the transmittance before irradiation, and $\alpha_{D}$ is the radiation induced absorption coefficient:

$$
\alpha_{D}=\alpha_{s a t}\left(1-e^{-\beta D}\right)+K D
$$

where $\alpha_{\text {sat }}$ ("saturation" value of $\alpha$ ), $\beta$, and $\mathrm{K}$ are independent of dose but depend on material and wavelength. (Treadaway 1975a,b; Levy 1985)

The term with $\alpha_{\text {sat }}$ and $\beta$ describes absorption due to the population of inherent defect sites. The term with $\mathrm{K}$ (linear in $\mathrm{D}$ ) describes absorption assoçiated with the formation of new defect sites (ion displacement). This term can generally be ignored for doses $<10^{8} \mathrm{rad}$. (Coakley 1992) However, for small doses $(D<<1 / \beta)$ the exponential in (Eq. 2) can be expanded to first order in $D$ and the dose dependence appears to be linear even though the term with coefficient $\mathrm{K}$ is negligible:

$$
\alpha_{D} \approx \beta \alpha_{\text {sat }} D \quad[D<<1 / \beta]
$$




\section{STABILIZED AND UNSTABILIZED GLASSES}

Optical glasses are non-crystalline, inorganic mixtures of various metallic oxides, fused by heating with materials such as silica or boric or phosphoric oxides. The oxides are blended to vary the refractive indices and alter the dispersion, to produce glasses with various desired optical properties. Unless stabilized by the addition of cerium, optical glasses tend to be noticeably darkened by doses of a few krad and may become essentially opaque to ultraviolet and visible radiation with doses of a few hundred krad. ${ }^{1}$

Multi-valence cerium ions can accept either electrons or holes and so the addition of cerium to glasses inhibits trapping of electrons or holes at defect sites and stabilizes the glass against darkening by ionizing radiation. According to (Stroud 1966) $\mathrm{Ce}^{3+}$ ions capture holes to form $\mathrm{Ce}^{3++}$ centers and Ce $\mathrm{C}^{4+}$ ions capture electrons to form ( $\mathrm{Ce}^{4+}+$ electron) centers. (Stroud includes an extensive list of references on radiation induced color centers in glasses.)

There is a penalty to pay for this stabilization: the cerium ions themselves act as color centers and cause the stabilized glasses to be more absorptive than ordinary glasses. This penalty is large for ultraviolet and short visible wavelengths but usually small for intermediate visible and infrared wavelengths. After radiation exposure, the ordinary glasses become more absorptive at all wavelengths whereas the stabilized glasses tend to retain their transmittance at visible and infrared wavelengths.

Information on stabilized and unstablized glasses can be found in the Schott Optical Glass catalogs listed in the references: (Schott c1980 and Schott c1990).

\section{THE STARTRACKER GLASSES}

We have explored the effects of radiation on two Startracker lens designs. One design uses unstabilized glasses; the other is a radiation stabilized design. Table 0 summarizes the arrangement of optical elements in the two designs. The optical elements were simulated using optical flats of the same glasses and (except for the LC fiber optic elements) with thicknesses equal the axial thicknesses of the elements in the lens system.

\footnotetext{
${ }^{1}$ Other metallic ions may be used instead of ccrium. Stroud (Stroud 1966) notes that iron, manganesc, and titanium also may stabilize glasses against radiation induced darkening. Cerium is, however, usually used for this purpose by the makers of optical glasses.
} 
Table 0. Arrangement of Optical Elements in the Startracker Lens

\begin{tabular}{|c|c|c|}
\hline $\begin{array}{l}\text { Element } \\
\text { Number }\end{array}$ & $\begin{array}{c}\text { Description } \\
\text { (Glass Type/Manufacturer) }\end{array}$ & $\begin{array}{c}\text { Axial } \\
\text { Thickness (cm) }\end{array}$ \\
\hline \multicolumn{3}{|c|}{ Unstabilized Design } \\
\hline $\begin{array}{l}1 \\
2 \\
3 \\
4 \\
5 \\
6\end{array}$ & $\begin{array}{l}\text { SFL 03/Ohara Optical Glass } \\
\text { SSK 4/Schott Optical Glass } \\
\text { SSK 4/Schott Optical Glass } \\
\text { LaF 20/Schott Optical Glass } \\
\text { LC }^{*} / \text { Gallileo Electro-Optic } \\
\text { RWY } 47^{*} / \text { Schott Fiber Optic }\end{array}$ & $\begin{array}{l}0.604 \\
0.556 \\
0.556 \\
0.604 \\
0.135 \\
0.7 \\
\end{array}$ \\
\hline \multicolumn{3}{|c|}{ Stabilized Design } \\
\hline $\begin{array}{l}1 \\
2 \\
3 \\
4 \\
5\end{array}$ & $\begin{array}{l}\text { SF } 6 \text { G5/Schott Optical Glass } \\
\text { LaK } 9 \text { G15/Schott Optical Glass } \\
\text { LaK } 9 \text { G15/Schott Optical Glass } \\
\text { LC*(Ce doped)/Gallileo Electro-Optic } \\
\text { RWY } 47 \text { (Ce doped)*/Schott Fiber Optic }\end{array}$ & $\begin{array}{c}0.825 \\
0.511 \\
1.34 \\
0.135 \\
0.7 \\
\end{array}$ \\
\hline
\end{tabular}

\section{THE EXPERIMENTS}

For the unstablized design, optical flats of thickness $.604 \mathrm{~cm}$ simulated the elements of SFL 03 and LaF 20. A single flat of thickness $1.11 \mathrm{~cm}$ sumulated the two elements of SSK 4. Fiber optic elements were simulated with flats of fiber optic material: $0.17 \mathrm{~cm}$ for the LC and $0.7 \mathrm{~cm}$ for the RWY 47 .

For the stabilized design, the SF 6 G5 element was simulated by an optical flat $0.825 \mathrm{~cm}$ thick. A single flat of thickness $1.85 \mathrm{~cm}$ simulated the two elements of LaK $9 \mathrm{G} 15$. Again, the fiber optic elements were simulated with flats of fiber optic material: $0.17 \mathrm{~cm}$ for the Ce doped LC and $0.7 \mathrm{~cm}$ for the Ce doped RWY 47.

We exposed the optical flats to ionizing radiation in LLNL's Co60 pool in the Standards and Calibration Laboratory. The report: "Calibration of the Standards and Calibration Laboratory's Co 60 Radiation Pool" (Wirtenson/White 1993) describes the facility and the experimental arrangements in more detail than is given here. Pursuant to the recommendations in (Wirtenson/White 1993), exposures were verified using opti-chromic dosimeters that were placed near each exposed sample. 
Each sample was exposed to 6 different doses. The doses ranged from $0.44 \mathrm{krad}$ to $1300 \mathrm{krad}$ but the set of doses varied from sample to sample.

After each exposure, the transmittance of each flat and of the combination of flats assembled in the Startracker configurations were measured at wavelengths ranging from $350 \mathrm{~nm}$ to $1200 \mathrm{~nm}$. Transmittance measurements were done with two instruments.:

1) A Cary 2300 Spectrophotometer (hereafter called "the Cary") was used with two lamp soirces: a deuterium source for the UV wavelengths and a tungsten-halogen source for thi: visible and near infrared wavelengths.

2) A Schœffel High Intensity Grating Moriochromator was used in conjunction with the Laser Precision RK5720 Power Ratiometer (hereafter called "the Ratiometer").

The Cary does not permit the required contact between fiber optic face plates and the photodetectors and therefore both the fiber optics and the assembled "lens" were measured only on the Ratiometer. The range of wavelengths probed by the Ratiometer is less than for the Cary and the Ratiometer data tended to be noisy and sometimes erratic so the Cary measurements were used in all determinations of the absorption parameters excepting those for the fiber optics.

The data so sbtained were fit, by least squares techniques, to obtain values for $T_{0}$ and the radiation induced absorption parameters $\alpha_{\text {sat }}$ and $\beta$ appearing in Eq. 2. These values of $T_{0}, \alpha_{\text {sat }}$ and $\beta$ were then used in Eqs. 1 and 2 (with $K=0$ ) to reconstruct the transmittance as a function of wavelength and dose.

Examination of plots of $\alpha_{\text {sat }}$ versus wavelength $(\lambda)$ for the unstabilized glasses suggested that a simple analytic relation:

$$
\alpha_{\text {sat }}=A e^{-k \lambda}
$$

fits the data well over a large range of wavelengths. Further, though the values of $\beta$ showed some dependence upon wavelength, they can, in most cases, be replaced by constants and still produce reasonably accurate fits to the transmittances.

The analytic form for $\alpha_{\text {sat }}$ (Eq. 4) did not work well for some of the stabilized glasses. Nevertheless, we did find some simple analytic approximations. The SF 6 G5 data can be broken into two wavelength ranges: < and > $630 \mathrm{~nm}$ and expressions of the form of (Eq. 4) with different parameters in each range give reasonably good fits to the transmittances. For the La K 9 G15 we found that the form of (Eq. 4 ) but with $\lambda$ replaced by $1 / \lambda$ works well. In all cases, the analytic fits may be poor where the transmittance is so low that the glass is probably of no interest as an optical transmitter. The form of (Eq. 4 )worked well for the Ce doped RWY 47 but a piecewise linear fit to b was found to be required to produce reasonably accurate fits to the transmittances using (Eqs. 1 and 2).

We have not estimated errors in the fit values of $T_{0}, \alpha_{\text {sat }}$ and $\beta$. We caution that the probable errors in $\alpha_{\text {sat }}$ and $\beta$ (but not $\mathrm{T}_{0}$ ) may be large; considerable deviations from the quoted values will produce tolerably good fits to the measured transmittances, especially at longer wavelengths. This is because the 
radiation induced absorption is a small perturbation on the zero dose transmittance for longer wavelengths so the effect being meisured may be less than the precision of the measurea transmittances.

Tables $1 \mathrm{a}-9 \mathrm{a}$ summarize the radiation induced absorption parameters determined by least squares fitting the transmittance data. The last column in each table shows the root mean square error in transmittance, calculated using the given parameters, compared to measured values. In most cases the error is averaged for 7 different doses). Table $1 b-9 b$ give the same analysis but use analytic fits to $\alpha_{\text {sat }}$ and $\beta$.

Figures 1a through 9a show measured (dots or other symbols) and calculated (solid lines) transmittances. The calculated values are those using the "best fit" for $\alpha_{\text {sat }}$ and $\beta$ given in Tables 1a through 9a. Note that the zero dose transmittances used to calculate the solid curves are the least squares fit values rather than the measured values. Thus, even at zero dose, the curves and the dots need not coincide.

In addition to the Ohara SFL 03, measurements were done for two samples of Schott SFL 57, a glass with composition similar to the SFL 03 that has nearly identical optical characteristics to SFL 03. The SFL 03 and SFL 57 samples showed very similar radiation induced absorption characteristics. The difference between SFL 03 and the SFL 57 is about the same as the difference between the two runs on the SFL 57 samples. The data for these three samples is reported in Tables $2 a, 2 a^{\prime}$ and $2 a^{\prime \prime}$. Table $2 a^{\prime \prime \prime}$ reports values of $T_{0}, \alpha_{\text {sat, }}$ and $\beta$ obtained by fitting transmittances averaged for the three samples. The "\% error " colum in Table 2a"' compares the fit using these averaged parameters in Eqs. 2 and 3, with the average of the transmittances measured for the three samples. Table $2 b$ gives suggested analytic fits to $\alpha_{\text {sat }}$ and $\beta$ and reports the rms error of the fit using these values of $\alpha_{\text {sat }}$ and $\beta$ in Eq. 2 to the average of the measured transmittances for the three samples.

A sample of Schott BK 7, a common optical glass, was also subjeted to several different doses of radiation and then analyzed along with the Startracker glasses. The BK 7 sample was $1.1 \mathrm{~cm}$ thick.

Note that we report transmittance; this includes not only internal absorption but also reflection effects at both the inner and outer glass surfaces. In an assembled lens, the reflections are much less at the interior glass-glass interfaces than they are at air-glass interfaces as occured in our photometer measurements. The transmittances shown should not be confused with transmissivities. To deduce transmissivities, it is necessary to "back out" the reflection effects from the transmittance. These contribute a factor to the total transmittance of approximately

$$
\left[1-\left(\frac{n-1}{n+1}\right)^{2}\right]^{2}
$$

where $\mathrm{n}$ is the index of refraction. In general, $\mathrm{n}$ depends on wavelength. For practical purposes, the transmissivity for the materials reviewed here is unity at the longer plotted wavelengths, and the reflectance correction depends only weakly on wavelength, so the conversion of transmittances to transmissivities can be achieved by dividing all transmittances transmittance at long wavelengths. 


\section{Schott SSK 4}

Table 1a: Least Squares Fit to $T_{0}, \alpha_{\text {sat }}$ and $\beta$, Schott SSK 4

\begin{tabular}{|c|c|c|c|c|}
\hline$\lambda(\mathrm{nm})$ & $\mathrm{T}_{0}$ & $\alpha_{\text {sat }}\left(\mathrm{cm}^{-1}\right)$ & $\beta\left(\mathrm{krad}^{-1}\right)$ & error $(\mathrm{ms} \%)$ \\
\hline 360 & $7.99 \mathrm{E}-01$ & $2.42 \mathrm{E}+01$ & $3.48 \mathrm{E}-03$ & $17.81 \%$ \\
400 & $8.63 \mathrm{E}-01$ & $1.87 \mathrm{E}+01$ & $3.70 \mathrm{E}-03$ & $6.42 \%$ \\
440 & $8.69 \mathrm{E}-01$ & $1.45 \mathrm{E}+01$ & $3.63 \mathrm{E}-03$ & $5.32 \%$ \\
480 & $8.78 \mathrm{E}-01$ & $1.13 \mathrm{E}+01$ & $3.38 \mathrm{E}-03$ & $3.95 \%$ \\
520 & $8.83 \mathrm{E}-01$ & $8.81 \mathrm{E}+00$ & $3.06 \mathrm{E}-03$ & $2.92 \%$ \\
560 & $8.87 \mathrm{E}-01$ & $6.88 \mathrm{E}+00$ & $2.84 \mathrm{E}-03$ & $2.18 \%$ \\
600 & $8.90 \mathrm{E}-01$ & $5.46 \mathrm{E}+00$ & $2.69 \mathrm{E}-03$ & $1.69 \%$ \\
680 & $8.94 \mathrm{E}-01$ & $3.22 \mathrm{E}+00$ & $2.82 \mathrm{E}-03$ & $1.06 \%$ \\
760 & $8.96 \mathrm{E}-01$ & $1.97 \mathrm{E}+00$ & $2.81 \mathrm{E}-03$ & $0.62 \%$ \\
840 & $8.97 \mathrm{E}-01$ & $1.15 \mathrm{E}+00$ & $2.75 \mathrm{E}-03$ & $0.30 \%$ \\
920 & $8.97 \mathrm{E}-01$ & $6.80 \mathrm{E}-01$ & $2.78 \mathrm{E}-03$ & $0.24 \%$ \\
1000 & $8.96 \mathrm{E}-01$ & $4.01 \mathrm{E}-01$ & $2.90 \mathrm{E}-03$ & $0.16 \%$ \\
1100 & $8.96 \mathrm{E}-01$ & $2.11 \mathrm{E}-01$ & $2.79 \mathrm{E}-03$ & $0.16 \%$ \\
1200 & $8.97 \mathrm{E}-01$ & $1.23 \mathrm{E}-01$ & $2.85 \mathrm{E}-03$ & $0.13 \%$ \\
\hline
\end{tabular}

Table 1b: Analytic Approximations for $\alpha_{\text {sat }}$ and $\beta$, Schott SSK 4 $\alpha_{\text {sat }}=240.31 \exp (-.0063586 \lambda) \mathrm{cm}^{-1}, \beta=.003 \mathrm{krad}^{-1}$

\begin{tabular}{|c|c|c|c|c|}
\hline$\lambda(\mathrm{nm})$ & $\mathrm{T}_{0}$ & $\alpha_{\text {sat }}(\mathrm{cm}-1)$ & $\beta\left(\mathrm{krad}^{-1}\right)$ & error $(\mathrm{rms} \%)$ \\
\hline 360 & $7.99 \mathrm{E}-01$ & $2.44 \mathrm{E}+01$ & $3.00 \mathrm{E}-03$ & $29.24 \%$ \\
400 & $8.63 \mathrm{E}-01$ & $1.89 \mathrm{E}+01$ & $3.00 \mathrm{E}-03$ & $47.28 \%$ \\
440 & $8.69 \mathrm{E}-01$ & $1.46 \mathrm{E}+01$ & $3.00 \mathrm{E}-03$ & $18.13 \%$ \\
480 & $8.78 \mathrm{E}-01$ & $1.14 \mathrm{E}+01$ & $3.00 \mathrm{E}-03$ & $9.19 \%$ \\
520 & $8.83 \mathrm{E}-01$ & $8.81 \mathrm{E}+00$ & $3.00 \mathrm{E}-03$ & $3.36 \%$ \\
560 & $8.87 \mathrm{E}-01$ & $6.83 \mathrm{E}+00$ & $3.00 \mathrm{E}-03$ & $3.05 \%$ \\
600 & $8.90 \mathrm{E}-01$ & $5.30 \mathrm{E}+00$ & $3.00 \mathrm{E}-03$ & $5.17 \%$ \\
680 & $8.94 \mathrm{E}-01$ & $3.18 \mathrm{E}+00$ & $3.00 \mathrm{E}-03$ & $1.55 \%$ \\
760 & $8.96 \mathrm{E}-01$ & $1.91 \mathrm{E}+00$ & $3.00 \mathrm{E}-03$ & $2.12 \%$ \\
840 & $8.97 \mathrm{E}-01$ & $1.15 \mathrm{E}+00$ & $3.00 \mathrm{E}-03$ & $0.81 \%$ \\
920 & $8.97 \mathrm{E}-01$ & $6.92 \mathrm{E}-01$ & $3.00 \mathrm{E}-03$ & $0.80 \%$ \\
1000 & $8.96 \mathrm{E}-01$ & $4.16 \mathrm{E}-01$ & $3.00 \mathrm{E}-03$ & $0.70 \%$ \\
1100 & $8.96 \mathrm{E}-01$ & $2.20 \mathrm{E}-01$ & $3.00 \mathrm{E}-03$ & $0.46 \%$ \\
1200 & $8.97 \mathrm{E}-01$ & $1.17 \mathrm{E}-01$ & $3.00 \mathrm{E}-03$ & $0.27 \%$ \\
\hline
\end{tabular}




\section{Schott SSK 4}

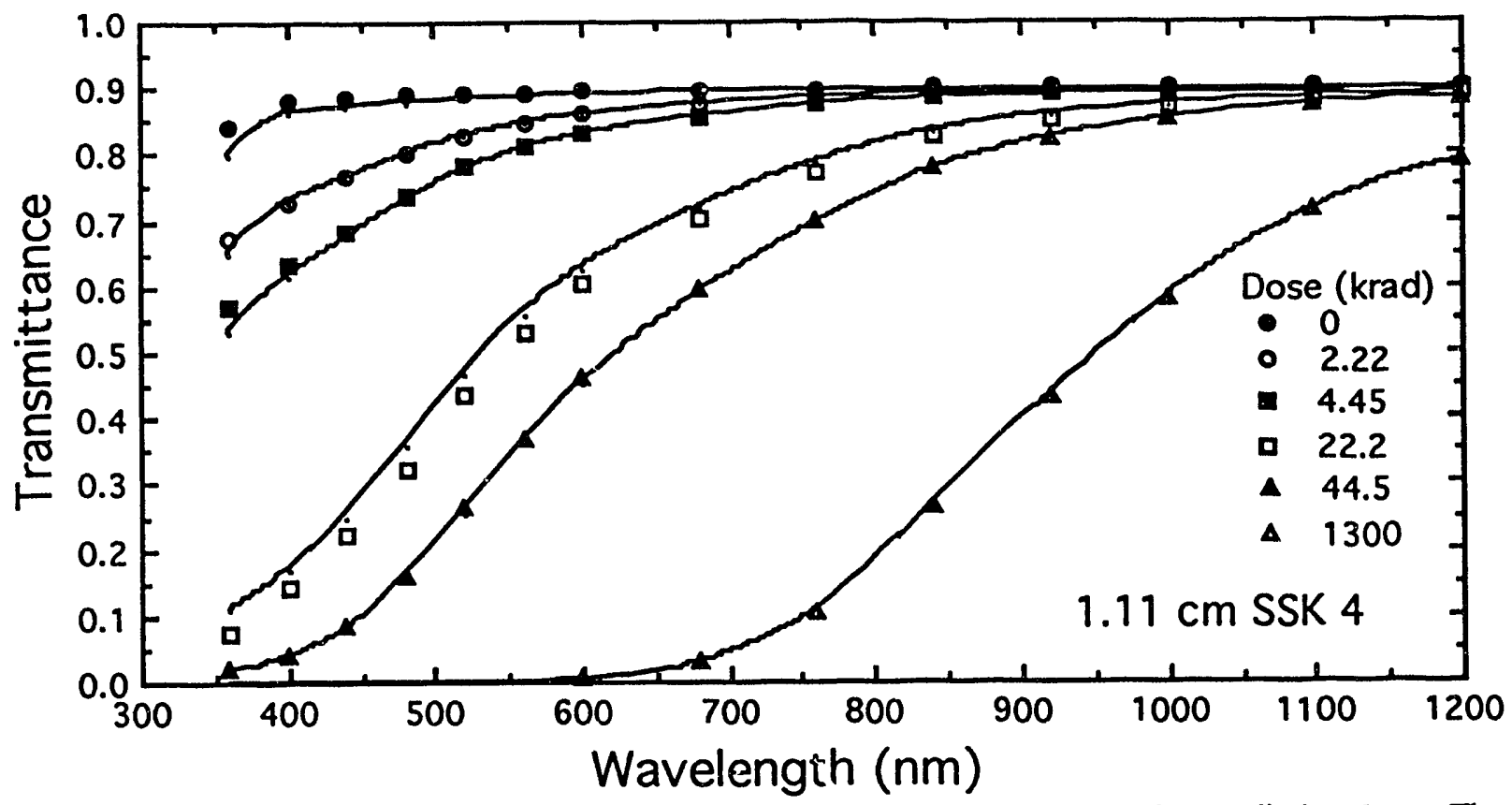

Fig. 1a: Transmittance vs. wavelength for $1.11 \mathrm{~cm}$ of Schot SSK 4 aiter exposure to various radiation doses. The plotted points are measured data; the solid lines are the fits using the "best fit" values to the radiation induced absorption parameters appearing in Eqs. 1 and 2. (The "best fit" values are shown as dots in the plots below.)
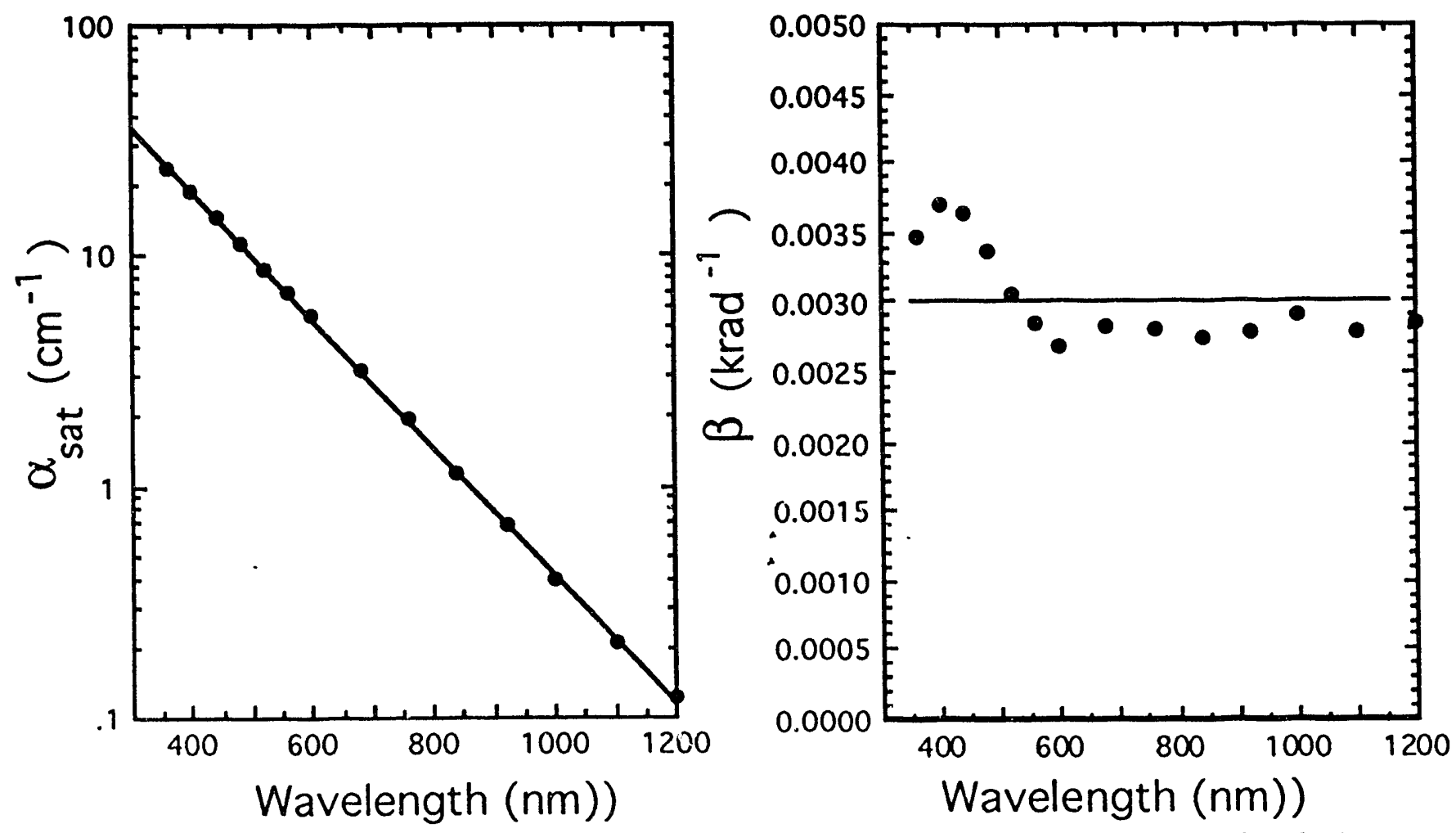

Fig. lb: Radiation Induced Absorption Parameters vs. Wavelength for Schott SSK 4. Dots are values given by least squares fits, solid lines are suggested analytic approximations to the fitted data. 
Schott SFL 57

Table 2a: Least Squares Fit to $\mathrm{T}_{0}, \alpha_{\text {sat }}$ and $\beta$, Schott SFL 57 Sample \#1

\begin{tabular}{|c|c|c|c|c|}
\hline$\lambda(\mathrm{nm})$ & $\mathrm{T}_{0}$ & $\alpha_{\text {sat }}\left(\mathrm{cm}^{-1}\right)$ & $\beta\left(\mathrm{krad}^{-1}\right)$ & error $(\mathrm{rms} \%)$ \\
\hline 380 & $1.59 \mathrm{E}-01$ & $1.64 \mathrm{E}+00$ & $1.95 \mathrm{E}-02$ & $11.67 \%$ \\
400 & $5.70 \mathrm{E}-01$ & $2.00 \mathrm{E}+00$ & $1.41 \mathrm{E}-02$ & $2.48 \%$ \\
440 & $7.64 \mathrm{E}-01$ & $1.78 \mathrm{E}+00$ & $1.45 \mathrm{E}-02$ & $1.49 \%$ \\
480 & $8.00 \mathrm{E}-01$ & $1.50 \mathrm{E}+00$ & $1.43 \mathrm{E}-02$ & $1.29 \%$ \\
520 & $8.18 \mathrm{E}-01$ & $1.18 \mathrm{E}+00$ & $1.38 \mathrm{E}-02$ & $1.01 \%$ \\
560 & $8.29 \mathrm{E}-01$ & $8.68 \mathrm{E}-01$ & $1.38 \mathrm{E}-02$ & $0.69 \%$ \\
600 & $8.34 \mathrm{E}-01$ & $6.28 \mathrm{E}-01$ & $1.32 \mathrm{E}-02$ & $0.64 \%$ \\
680 & $8.42 \mathrm{E}-01$ & $3.18 \mathrm{E}-01$ & $2.10 \mathrm{E}-02$ & $1.47 \%$ \\
760 & $8.31 \mathrm{E}-01$ & $1.25 \mathrm{E}-01$ & $8.00 \mathrm{E}-03$ & $0.93 \%$ \\
840 & $8.42 \mathrm{E}-01$ & $7.41 \mathrm{E}-02$ & $5.78 \mathrm{E}-03$ & $0.08 \%$ \\
920 & $8.42 \mathrm{E}-01$ & $4.36 \mathrm{E}-02$ & $3.27 \mathrm{E}-03$ & $0.11 \%$ \\
1000 & $8.42 \mathrm{E}-01$ & $2.43 \mathrm{E}-02$ & $2.32 \mathrm{E}-03$ & $0.14 \%$ \\
1100 & $8.42 \mathrm{E}-01$ & $1.84 \mathrm{E}-02$ & $9.68 \mathrm{E}-04$ & $0.15 \%$ \\
1200 & $8.42 \mathrm{E}-01$ & $1.14 \mathrm{E}+00$ & $3.05 \mathrm{E}-06$ & $0.21 \%$ \\
\hline
\end{tabular}

Table 2a': Least Squares Fit to $T_{0}, \alpha_{\text {sat }}$ and $\beta$, Schott SFL 57 Sample $\# 2$

\begin{tabular}{|c|c|c|c|c|}
\hline$\lambda(\mathrm{nm})$ & $\mathrm{T}_{0}$ & $\alpha_{\text {sat }}\left(\mathrm{cm}^{-1}\right)$ & $\beta\left(\mathrm{krad}^{-1}\right)$ & error $(\mathrm{rms} \%)$ \\
\hline 380 & $1.43 \mathrm{E}-01$ & $1.43 \mathrm{E}+00$ & $2.88 \mathrm{E}-02$ & $10.46 \%$ \\
400 & $5.62 \mathrm{E}-01$ & $1.96 \mathrm{E}+00$ & $1.65 \mathrm{E}-02$ & $2.89 \%$ \\
440 & $7.60 \mathrm{E}-01$ & $1.73 \mathrm{E}+00$ & $2.02 \mathrm{E}-02$ & $5.44 \%$ \\
480 & $8.05 \mathrm{E}-01$ & $1.50 \mathrm{E}+00$ & $1.66 \mathrm{E}-02$ & $1.61 \%$ \\
520 & $8.21 \mathrm{E}-01$ & $1.18 \mathrm{E}+00$ & $1.64 \mathrm{E}-02$ & $1.33 \%$ \\
560 & $8.32 \mathrm{E}-01$ & $8.71 \mathrm{E}-01$ & $1.64 \mathrm{E}-02$ & $1.02 \%$ \\
600 & $8.35 \mathrm{E}-01$ & $6.29 \mathrm{E}-01$ & $1.62 \mathrm{E}-02$ & $0.76 \%$ \\
680 & $8.39 \mathrm{E}-01$ & $2.98 \mathrm{E}-01$ & $1.69 \mathrm{E}-02$ & $0.51 \%$ \\
760 & $8.45 \mathrm{E}-01$ & $1.52 \mathrm{E}-01$ & $1.64 \mathrm{E}-02$ & $0.31 \%$ \\
840 & $8.47 \mathrm{E}-01$ & $8.37 \mathrm{E}-02$ & $7.72 \mathrm{E}-03$ & $0.22 \%$ \\
920 & $8.48 \mathrm{E}-01$ & $5.49 \mathrm{E}-02$ & $6.80 \mathrm{E}-03$ & $0.20 \%$ \\
1000 & $8.48 \mathrm{E}-01$ & $3.61 \mathrm{E}-02$ & $7.95 \mathrm{E}-03$ & $0.24 \%$ \\
1100 & $8.50 \mathrm{E}-01$ & $2.90 \mathrm{E}-02$ & $9.70 \mathrm{E}-03$ & $0.25 \%$ \\
1200 & $8.50 \mathrm{E}-01$ & $2.07 \mathrm{E}-02$ & $1.07 \mathrm{E}-02$ & $0.23 \%$ \\
\hline
\end{tabular}




\section{Ohara SFL 03}

Table 2a": Least Squares Fit to $T_{0}, \alpha_{\text {sat }}$ and $\beta$, Ohara SFL 03

\begin{tabular}{|c|c|c|c|c|}
\hline$\lambda(\mathrm{nm})$ & $\mathrm{T}_{0}$ & $\alpha_{\text {sat }}\left(\mathrm{cm}^{-1}\right)$ & $\beta\left(\mathrm{krad}^{-1}\right)$ & error (ms\%) \\
\hline 380 & $1.66 \mathrm{E}-01$ & $1.74 \mathrm{C}+00$ & $1.20 \mathrm{E}-02$ & $1.87 \%$ \\
400 & $6.11 \mathrm{E}-01$ & $2.11 \mathrm{E}+00$ & $1.24 \mathrm{E}-02$ & $1.88 \%$ \\
440 & $7.81 \mathrm{E}-01$ & $1.88 \mathrm{E}+00$ & $1.31 \mathrm{E}-02$ & $2.24 \%$ \\
60 & $8.06 \mathrm{E}-01$ & $1.58 \mathrm{E}+00$ & $1.30 \mathrm{E}-02$ & $1.92 \%$ \\
520 & $8.20 \mathrm{E}-01$ & $1.26 \mathrm{E}+00$ & $1.26 \mathrm{E}-02$ & $1.55 \%$ \\
560 & $8.30 \mathrm{E}-01$ & $9.37 \mathrm{E}-01$ & $1.23 \mathrm{E}-02$ & $1.11 \%$ \\
600 & $8.34 \mathrm{E}-01$ & $6.87 \mathrm{E}-01$ & $1.18 \mathrm{E}-02$ & $0.82 \%$ \\
680 & $8.38 \mathrm{E}-01$ & $3.94 \mathrm{E}-01$ & $8.92 \mathrm{E}-03$ & $0.44 \%$ \\
760 & $8.44 \mathrm{E}-01$ & $1.74 \mathrm{E}-01$ & $1.06 \mathrm{E}-02$ & $0.09 \%$ \\
840 & $8.44 \mathrm{E}-01$ & $7.88 \mathrm{E}-02$ & $6.16 \mathrm{E}-03$ & $0.43 \%$ \\
920 & $8.44 \mathrm{E}-01$ & $4.13 \mathrm{E}-02$ & $3.78 \mathrm{E}-03$ & $0.46 \%$ \\
1000 & $8.44 \mathrm{E}-01$ & $1.71 \mathrm{E}-02$ & $8.66 \mathrm{E}-03$ & $0.46 \%$ \\
1100 & $8.51 \mathrm{E}-01$ & $1.81 \mathrm{E}-02$ & $1.63 \mathrm{E}+00$ & $0.31 \%$ \\
1200 & $8.44 \mathrm{E}-01$ & $-8.21 \mathrm{E}-03$ & $1.11 \mathrm{E}-02$ & $0.52 \%$ \\
\hline
\end{tabular}


Average of 1 Sample Ohara SFL 03 and 2 Samples of Schott SFL 57

Table 2a"': Least Squares Fit to $\mathrm{T}_{0}, \alpha_{\text {sat }}$ and $\beta$ (averaged data) (Fits are to T's averaged for the two SFL 57 samples and one SFL 03 sample)

\begin{tabular}{|c|c|c|c|c|}
\hline$\lambda(\mathrm{nm})$ & $\mathrm{T}_{0}$ & $\alpha_{3 \text { at }}\left(\mathrm{cm}^{-1}\right)$ & $\beta\left(\mathrm{krad}^{-1}\right)$ & error $(\mathrm{ms} \%)$ \\
\hline 380 & $1.80 \mathrm{E}-01$ & $1.61 \mathrm{E}+00$ & $2.01 \mathrm{E}-02$ & $14.52 \%$ \\
400 & $5.98 \mathrm{E}-01$ & $2.02 \mathrm{E}+00$ & $1.43 \mathrm{E}-02$ & $3.59 \%$ \\
440 & $7.83 \mathrm{E}-01$ & $1.80 \mathrm{E}+00$ & $1.59 \mathrm{E}-02$ & $3.47 \%$ \\
480 & $8.14 \mathrm{E}-01$ & $1.52 \mathrm{E}+00$ & $1.46 \mathrm{E}-02$ & $2.00 \%$ \\
520 & $8.28 \mathrm{E}-01$ & $1.21 \mathrm{E}+00$ & $1.43 \mathrm{E}-02$ & $1.61 \%$ \\
560 & $8.36 \mathrm{E}-01$ & $8.92 \mathrm{E}-01$ & $1.42 \mathrm{E}-02$ & $1.10 \%$ \\
600 & $8.37 \mathrm{E}-01$ & $6.48 \mathrm{E}-01$ & $1.37 \mathrm{E}-02$ & $0.74 \%$ \\
680 & $8.40 \mathrm{E}-01$ & $3.37 \mathrm{E}-01$ & $1.56 \mathrm{E}-02$ & $0.33 \%$ \\
760 & $8.44 \mathrm{E}-01$ & $1.63 \mathrm{E}-01$ & $1.35 \mathrm{E}-02$ & $0.20 \%$ \\
840 & $8.48 \mathrm{E}-01$ & $7.89 \mathrm{E}-02$ & $6.55 \mathrm{E}-03$ & $0.49 \%$ \\
920 & $8.48 \mathrm{E}-01$ & $4.66 \mathrm{E}-02$ & $4.62 \mathrm{E}-03$ & $0.53 \%$ \\
1000 & $8.49 \mathrm{E}-01$ & $2.58 \mathrm{E}-02$ & $6.31 \mathrm{E}-03$ & $0.57 \%$ \\
1100 & $8.49 \mathrm{E}-01$ & $1.09 \mathrm{E}-02$ & $5.48 \mathrm{E}-01$ & $0.36 \%$ \\
1200 & $8.50 \mathrm{E}-01$ & $4.91 \mathrm{E}-03$ & $7.27 \mathrm{E}-03$ & $0.61 \%$ \\
\hline
\end{tabular}

Table 2b: Analytic Approximations for $\alpha_{\text {sat }}$ and $\beta$, Schott SFL 57 and Ohara SFL 03 (Fits are to T's averaged for the two SFL 57 samples and one SFL 03 sample)

\begin{tabular}{|c|c|c|c|c|}
\multicolumn{5}{c|}{$\alpha_{\text {sat }}=240.31 \exp (-.007988 \lambda) \mathrm{cm}^{-1}, \beta=.0146 \mathrm{krad}^{-1}$} \\
\hline$\lambda(\mathrm{nm})$ & $\mathrm{T}_{0}$ & $\alpha_{\text {sat }}\left(\mathrm{cm}^{-1}\right)$ & $\beta\left(\mathrm{krad}^{-1}\right)$ & error $(\mathrm{ms} \%)$ \\
\hline 380 & $1.80 \mathrm{E}-01$ & $3.44 \mathrm{E}+00$ & $1.46 \mathrm{E}-02$ & $25.29 \%$ \\
400 & $5.98 \mathrm{E}-01$ & $2.93 \mathrm{E}+00$ & $1.46 \mathrm{E}-02$ & $24.39 \%$ \\
440 & $7.83 \mathrm{E}-01$ & $2.13 \mathrm{E}+00$ & $1.46 \mathrm{E}-02$ & $8.27 \%$ \\
480 & $8.14 \mathrm{E}-01$ & $1.55 \mathrm{E}+00$ & $1.46 \mathrm{E}-02$ & $1.86 \%$ \\
520 & $8.28 \mathrm{E}-01$ & $1.12 \mathrm{E}+00$ & $1.46 \mathrm{E}-02$ & $3.19 \%$ \\
560 & $8.36 \mathrm{E}-01$ & $8.16 \mathrm{E}-01$ & $1.46 \mathrm{E}-02$ & $2.59 \%$ \\
600 & $8.37 \mathrm{E}-01$ & $5.93 \mathrm{E}-01$ & $1.46 \mathrm{E}-02$ & $1.69 \%$ \\
680 & $8.40 \mathrm{E}-01$ & $3.13 \mathrm{E}-01$ & $1.46 \mathrm{E}-02$ & $0.68 \%$ \\
760 & $8.44 \mathrm{E}-01$ & $1.65 \mathrm{E}-01$ & $1.46 \mathrm{E}-02$ & $0.37 \%$ \\
840 & $8.48 \mathrm{E}-01$ & $8.71 \mathrm{E}-02$ & $1.46 \mathrm{E}-02$ & $0.60 \%$ \\
920 & $8.48 \mathrm{E}-01$ & $4.60 \mathrm{E}-02$ & $1.46 \mathrm{E}-02$ & $0.47 \%$ \\
1000 & $8.49 \mathrm{E}-01$ & $2.43 \mathrm{E}-02$ & $1.46 \mathrm{E}-02$ & $0.51 \%$ \\
1100 & $8.49 \mathrm{E}-01$ & $1.09 \mathrm{E}-02$ & $1.46 \mathrm{E}-02$ & $0.59 \%$ \\
1200 & $8.50 \mathrm{E}-01$ & $4.91 \mathrm{E}-03$ & $1.46 \mathrm{E}-02$ & $0.60 \%$ \\
\hline
\end{tabular}


Ohara SFL 03 and Schott SFL 57

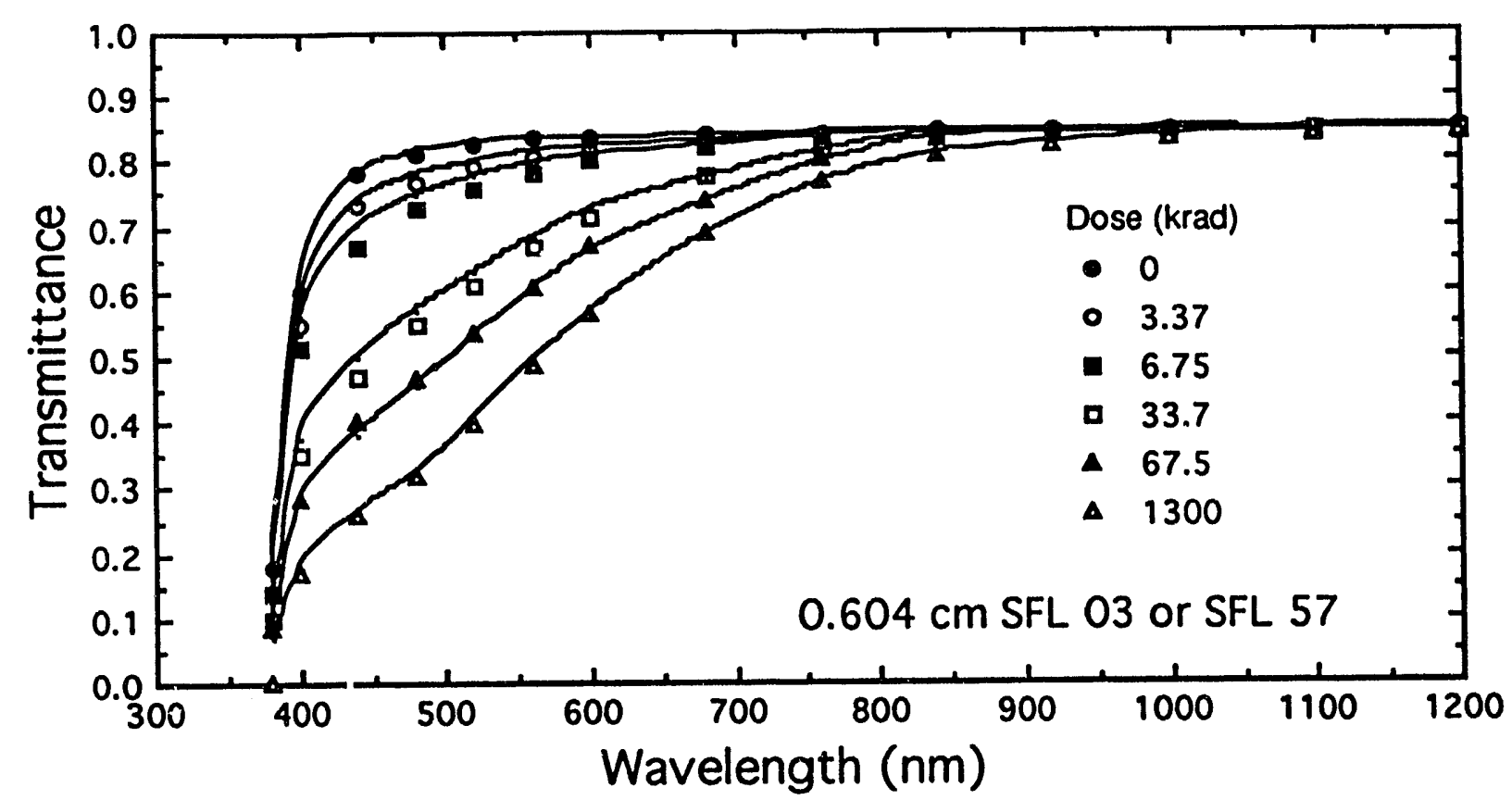

Fig. 2a: Transmittance (averaged for three samples) vs. wavelength for $0.604 \mathrm{~cm}$ of Schott SFL 57 (2 samples) and Ohara SFL 03 (1 sample) after exposure to various radiation doses. The plotted points are averaged measured data; the solid lines are the fits using the "best fit" values to the radiation induced absorption parameters appearing in Eqs. 1 and 2. (The "best fit" values are shown as dots in the plots below.)
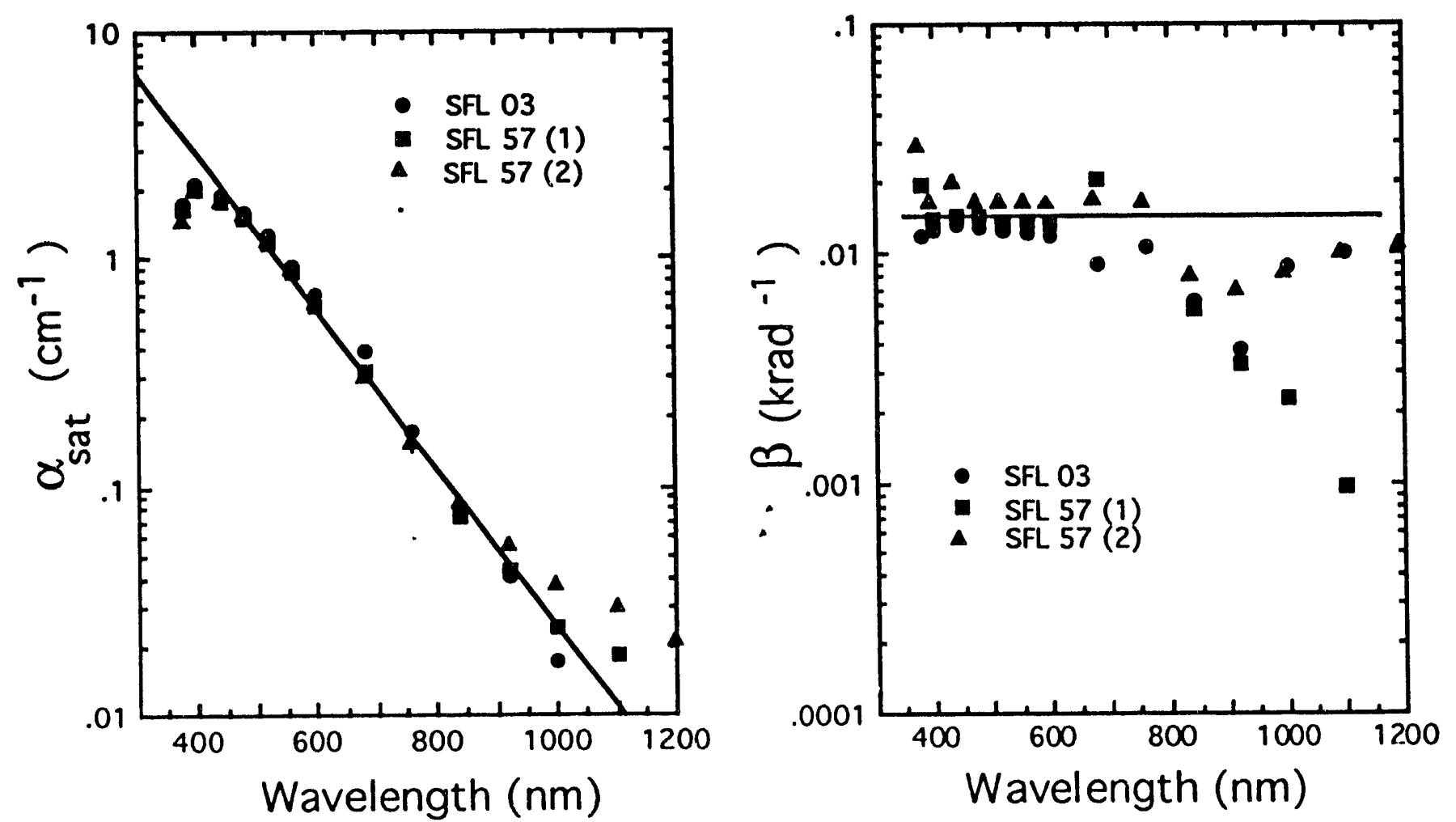

Fig. 2b: Kadiation Induced Absorption Parameters vs. Wavelength for Schou SFL 57 and Ohara SFL 03. Dots are values given by least squares fits, solid lines are suggested analytic approximations to the fitted data. 


\section{Schott LaF 20}

Table 3a: Least Squares Fit to $\mathrm{T}_{0}, \alpha_{\text {sat }}$ and $\beta$, Schott LaF 20

\begin{tabular}{|c|c|c|c|c|}
\hline$\lambda(\mathrm{nm})$ & $\mathrm{T}_{0}$ & $\alpha_{\text {sat }}\left(\mathrm{cm}^{-1}\right)$ & $\beta\left(\mathrm{krad}^{-1}\right)$ & error $(\mathrm{ms} \%)$ \\
\hline 360 & $5.47 \mathrm{E}-01$ & $9.02 \mathrm{E}+00$ & $3.45 \mathrm{E}-03$ & $3.79 \%$ \\
400 & $8.37 \mathrm{E}-01$ & $6.72 \mathrm{E}+00$ & $3.55 \mathrm{E}-03$ & $0.39 \%$ \\
440 & $8.67 \mathrm{E}-01$ & $5.03 \mathrm{E}+00$ & $3.41 \mathrm{E}-03$ & $0.31 \%$ \\
480 & $8.74 \mathrm{E}-01$ & $4.42 \mathrm{E}+00$ & $2.80 \mathrm{E}-03$ & $0.24 \%$ \\
520 & $8.78 \mathrm{E}-01$ & $3.09 \mathrm{E}+00$ & $3.11 \mathrm{E}-03$ & $0.21 \%$ \\
560 & $8.79 \mathrm{E}-01$ & $2.65 \mathrm{E}+00$ & $2.97 \mathrm{E}-03$ & $0.16 \%$ \\
600 & $8.80 \mathrm{E}-01$ & $2.31 \mathrm{E}+00$ & $2.91 \mathrm{E}-03$ & $0.15 \%$ \\
680 & $8.81 \mathrm{E}-01$ & $1.60 \mathrm{E}+00$ & $2.86 \mathrm{E}-03$ & $0.11 \%$ \\
760 & $8.82 \mathrm{E}-01$ & $9.64 \mathrm{E}-01$ & $2.85 \mathrm{E}-03$ & $0.06 \%$ \\
840 & $8.81 \mathrm{E}-01$ & $5.27 \mathrm{E}-01$ & $2.63 \mathrm{E}-03$ & $0.18 \%$ \\
920 & $8.81 \mathrm{E}-01$ & $2.99 \mathrm{E}-01$ & $2.55 \mathrm{E}-03$ & $0.13 \%$ \\
1000 & $8.81 \mathrm{E}-01$ & $1.65 \mathrm{E}-01$ & $3.35 \mathrm{E}-03$ & $0.14 \%$ \\
1100 & $8.81 \mathrm{E}-01$ & $9.92 \mathrm{E}-02$ & $4.10 \mathrm{E}-03$ & $0.09 \%$ \\
1200 & $8.81 \mathrm{E}-01$ & $6.46 \mathrm{E}-02$ & $3.68 \mathrm{E}-03$ & $0.06 \%$ \\
\hline
\end{tabular}

Table 3b: Analytic Approximations for $\alpha_{\text {sat }}$ and $\beta$, Schott LaF 20 $\alpha_{\text {sat }}=76.373 \exp (-.005966 \lambda) \mathrm{cm}^{-1}, \beta=.0032 \mathrm{krad}^{-1}$

\begin{tabular}{|c|c|c|c|c|}
\hline$\lambda(\mathrm{nm})$ & $\mathrm{T}_{0}$ & $\alpha_{\text {sat }}\left(\mathrm{cm}^{-1}\right)$ & $\beta\left(\mathrm{krad}^{-1}\right)$ & error $(\mathrm{ms} \%)$ \\
\hline 360 & $5.47 \mathrm{E}-01$ & $8.92 \mathrm{E}+00$ & $3.20 \mathrm{E}-03$ & $5.64 \%$ \\
400 & $8.37 \mathrm{E}-01$ & $7.02 \mathrm{E}+00$ & $3.20 \mathrm{E}-03$ & $6.03 \%$ \\
440 & $8.67 \mathrm{E}-01$ & $5.53 \mathrm{E}+00$ & $3.20 \mathrm{E}-03$ & $10.85 \%$ \\
480 & $8.74 \mathrm{E}-01$ & $4.36 \mathrm{E}+00$ & $3.20 \mathrm{E}-03$ & $1.64 \%$ \\
520 & $8.78 \mathrm{E}-01$ & $3.43 \mathrm{E}+00$ & $3.20 \mathrm{E}-03$ & $7.89 \%$ \\
560 & $8.79 \mathrm{E}-01$ & $2.70 \mathrm{E}+00$ & $3.20 \mathrm{E}-03$ & $1.65 \%$ \\
600 & $8.80 \mathrm{E}-01$ & $2.13 \mathrm{E}+00$ & $3.20 \mathrm{E}-03$ & $3.69 \%$ \\
680 & $8.81 \mathrm{E}-01$ & $1.32 \mathrm{E}+00$ & $3.20 \mathrm{E}-03$ & $5.98 \%$ \\
760 & $8.82 \mathrm{E}-01$ & $8.20 \mathrm{E}-01$ & $3.20 \mathrm{E}-03$ & $3.03 \%$ \\
840 & $8.81 \mathrm{E}-01$ & $5.09 \mathrm{E}-01$ & $3.20 \mathrm{E}-03$ & $0.36 \%$ \\
920 & $8.81 \mathrm{E}-01$ & $3.16 \mathrm{E}-01$ & $3.20 \mathrm{E}-03$ & $0.59 \%$ \\
1000 & $8.81 \mathrm{E}-01$ & $1.96 \mathrm{E}-01$ & $3.20 \mathrm{E}-03$ & $0.69 \%$ \\
1100 & $8.81 \mathrm{E}-01$ & $1.08 \mathrm{E}-01$ & $3.20 \mathrm{E}-03$ & $0.20 \%$ \\
1200 & $8.81 \mathrm{E}-01$ & $5.94 \mathrm{E}-02$ & $3.20 \mathrm{E}-03$ & $0.15 \%$ \\
\hline
\end{tabular}




\section{Schott LaF 20}

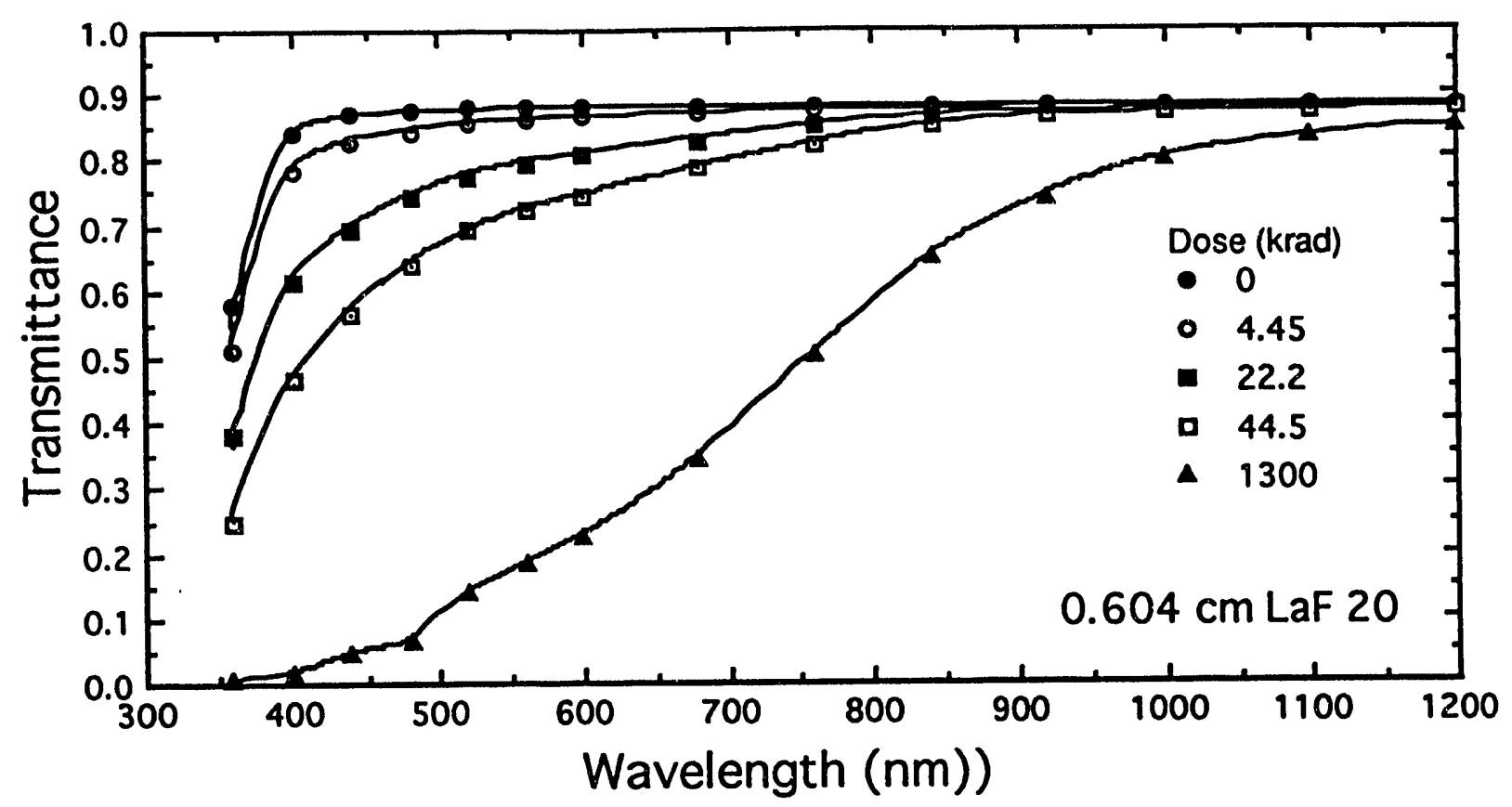

Fig. 3a: Transmittance vs. wavelength for $0.604 \mathrm{~cm}$ of Schott LaF 20 after exposure to various radiation doses. The plotted points are measured data; the solid lines are the fits using the "best fit" values to the radiation induced absorption parameters appearing in Eqs. 1 and 2. (The "best fit" values are shown as dots in the plots below.)
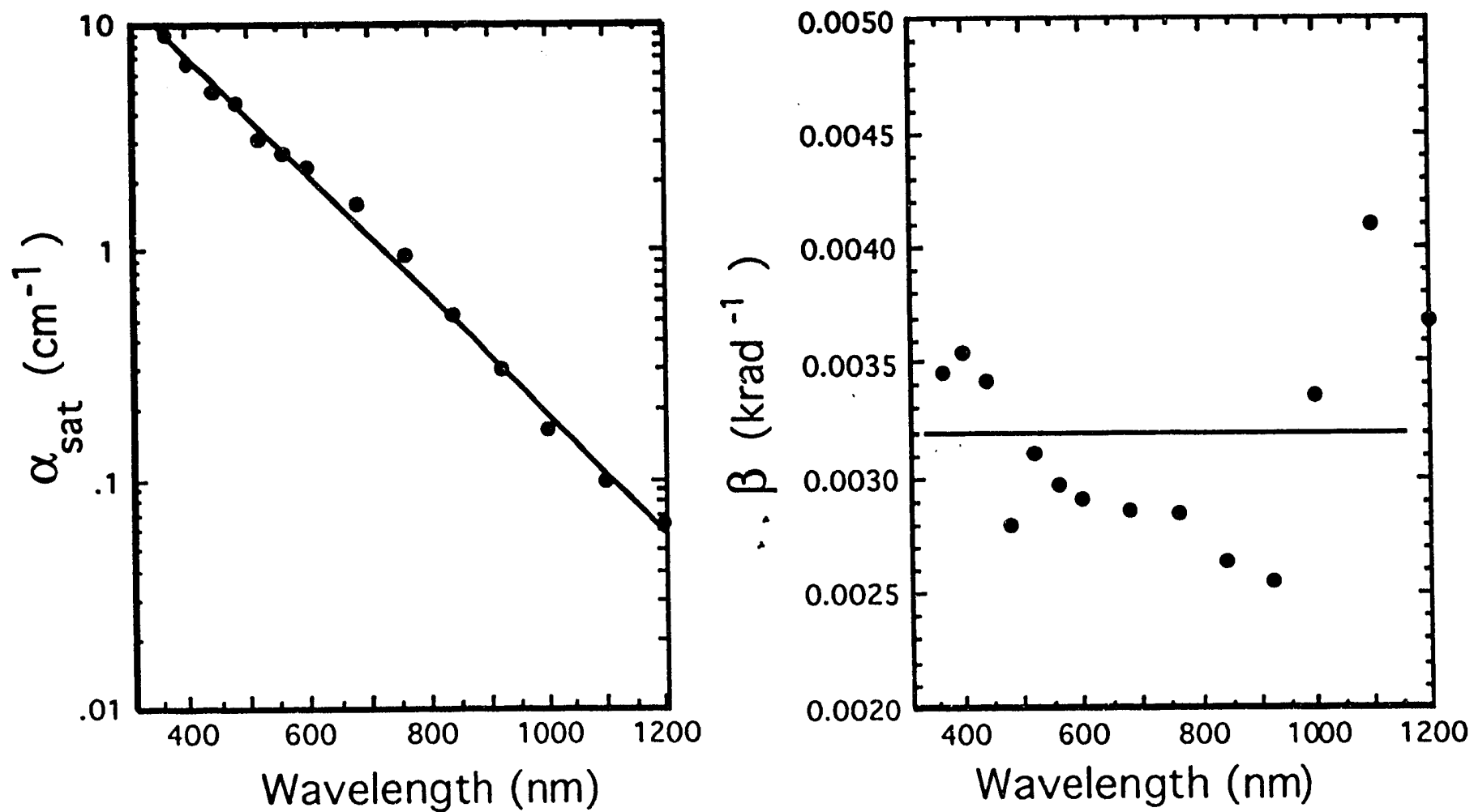

Fig. 3b: Radiation Induced Absorption Parameters vs. Wavelength for Schott LaF 20. Dots are values given by least squares fits, solid lines are suggested analytic approximations to the fitted data. 
Gallileo Electro-Optic LC Fiber Optics (undoped) Table 4a: Least Squares Fit to $T_{0}, \alpha_{\text {sat }}$ and $\beta$; Gallileo Electro-Optic LC Fiber Optics.

\begin{tabular}{|c|c|c|c|c|}
\hline$\lambda(\mathrm{nm})$ & $\mathrm{T}_{0}$ & $\alpha_{\text {sat }}\left(\mathrm{cm}^{-1}\right)$ & $\beta\left(\mathrm{krad}^{-1}\right)$ & error $(\mathrm{rms} \%)$ \\
\hline 362.79 & $2.44 \mathrm{E}-01$ & $1.43 \mathrm{E}+01$ & $2.43 \mathrm{E}-02$ & $2.05 \%$ \\
399.14 & $5.18 \mathrm{E}-01$ & $1.34 \mathrm{E}+01$ & $2.74 \mathrm{E}-02$ & $1.25 \%$ \\
422.27 & $6.22 \mathrm{E}-01$ & $1.36 \mathrm{E}+01$ & $2.87 \mathrm{E}-02$ & $1.15 \%$ \\
461.93 & $6.99 \mathrm{E}-01$ & $1.34 \mathrm{E}+01$ & $2.88 \mathrm{E}-02$ & $0.92 \%$ \\
501.58 & $7.26 \mathrm{E}-01$ & $1.23 \mathrm{E}+01$ & $2.78 \mathrm{E}-02$ & $0.85 \%$ \\
541.24 & $7.47 \mathrm{E}-01$ & $1.03 \mathrm{E}+01$ & $2.72 \mathrm{E}-02$ & $0.86 \%$ \\
580.89 & $7.59 \mathrm{E}-01$ & $8.28 \mathrm{E}+00$ & $2.61 \mathrm{E}-02$ & $0.66 \%$ \\
620.55 & $7.68 \mathrm{E}-01$ & $6.10 \mathrm{E}+00$ & $2.76 \mathrm{E}-02$ & $0.71 \%$ \\
640.37 & $7.70 \mathrm{E}-01$ & $5.28 \mathrm{E}+00$ & $2.84 \mathrm{E}-02$ & $0.73 \%$ \\
660.20 & $7.72 \mathrm{E}-01$ & $4.65 \mathrm{E}+00$ & $2.94 \mathrm{E}-02$ & $0.69 \%$ \\
676.72 & $7.70 \mathrm{E}-01$ & $4.47 \mathrm{E}+00$ & $2.74 \mathrm{E}-02$ & $0.68 \%$ \\
722.99 & $7.71 \mathrm{E}-01$ & $3.78 \mathrm{E}+00$ & $2.80 \mathrm{E}-02$ & $0.63 \%$ \\
762.64 & $7.76 \mathrm{E}-01$ & $3.14 \mathrm{E}+00$ & $3.05 \mathrm{E}-02$ & $0.55 \%$ \\
798.99 & $7.74 \mathrm{E}-01$ & $2.90 \mathrm{E}+00$ & $2.78 \mathrm{E}-02$ & $0.49 \%$ \\
\hline
\end{tabular}

Table 4b: Analytic Approximations for $\alpha_{\text {sat }}$ and $\beta$, Undoped LC Fiber Optics.

$\alpha_{\text {sat }}=156.52 \exp (-.0051440 \lambda) \mathrm{cm}^{-1}, \beta=.028 \mathrm{krad}^{-1}$

\begin{tabular}{|c|c|c|c|c|}
\hline$\lambda(\mathrm{nm})$ & $\mathrm{T}_{0}$ & $\alpha_{\text {sat }}\left(\mathrm{cm}^{-1}\right)$ & $\beta\left(\mathrm{krad}^{-1}\right)$ & error $(\mathrm{mms} \%)$ \\
\hline 362.79 & $2.44 \mathrm{E}-01$ & $2.42 \mathrm{E}+01$ & $2.80 \mathrm{E}-02$ & $79.92 \%$ \\
399.14 & $5.18 \mathrm{E}-01$ & $2.01 \mathrm{E}+01$ & $2.80 \mathrm{E}-02$ & $50.07 \%$ \\
422.27 & $6.22 \mathrm{E}-01$ & $1.78 \mathrm{E}+01$ & $2.80 \mathrm{E}-02$ & $30.42 \%$ \\
461.93 & $6.99 \mathrm{E}-01$ & $1.45 \mathrm{E}+01$ & $2.80 \mathrm{E}-02$ & $7.21 \%$ \\
501.58 & $7.26 \mathrm{E}-01$ & $1.19 \mathrm{E}+01$ & $2.80 \mathrm{E}-02$ & $2.71 \%$ \\
541.24 & $7.47 \mathrm{E}-01$ & $9.67 \mathrm{E}+00$ & $2.80 \mathrm{E}-02$ & $4.05 \%$ \\
580.89 & $7.59 \mathrm{E}-01$ & $7.89 \mathrm{E}+00$ & $2.80 \mathrm{E}-02$ & $1.36 \%$ \\
620.55 & $7.68 \mathrm{E}-01$ & $6.43 \mathrm{E}+00$ & $2.80 \mathrm{E}-02$ & $2.87 \%$ \\
640.37 & $7.70 \mathrm{E}-01$ & $5.81 \mathrm{E}+00$ & $2.80 \mathrm{E}-02$ & $3.68 \%$ \\
660.20 & $7.72 \mathrm{E}-01$ & $5.24 \mathrm{E}+00$ & $2.80 \mathrm{E}-02$ & $3.71 \%$ \\
676.72 & $7.70 \mathrm{E}-01$ & $4.82 \mathrm{E}+00$ & $2.80 \mathrm{E}-02$ & $2.98 \%$ \\
722.99 & $7.71 \mathrm{E}-01$ & $3.80 \mathrm{E}+00$ & $2.80 \mathrm{E}-02$ & $0.64 \%$ \\
762.64 & $7.76 \mathrm{E}-01$ & $3.10 \mathrm{E}+00$ & $2.80 \mathrm{E}-02$ & $1.34 \%$ \\
798.99 & $7.74 \mathrm{E}-01$ & $2.57 \mathrm{E}+00$ & $2.80 \mathrm{E}-02$ & $2.41 \%$ \\
\hline
\end{tabular}




\section{Gallileo Electro-Optic LC Fiber Optics (undoped)}

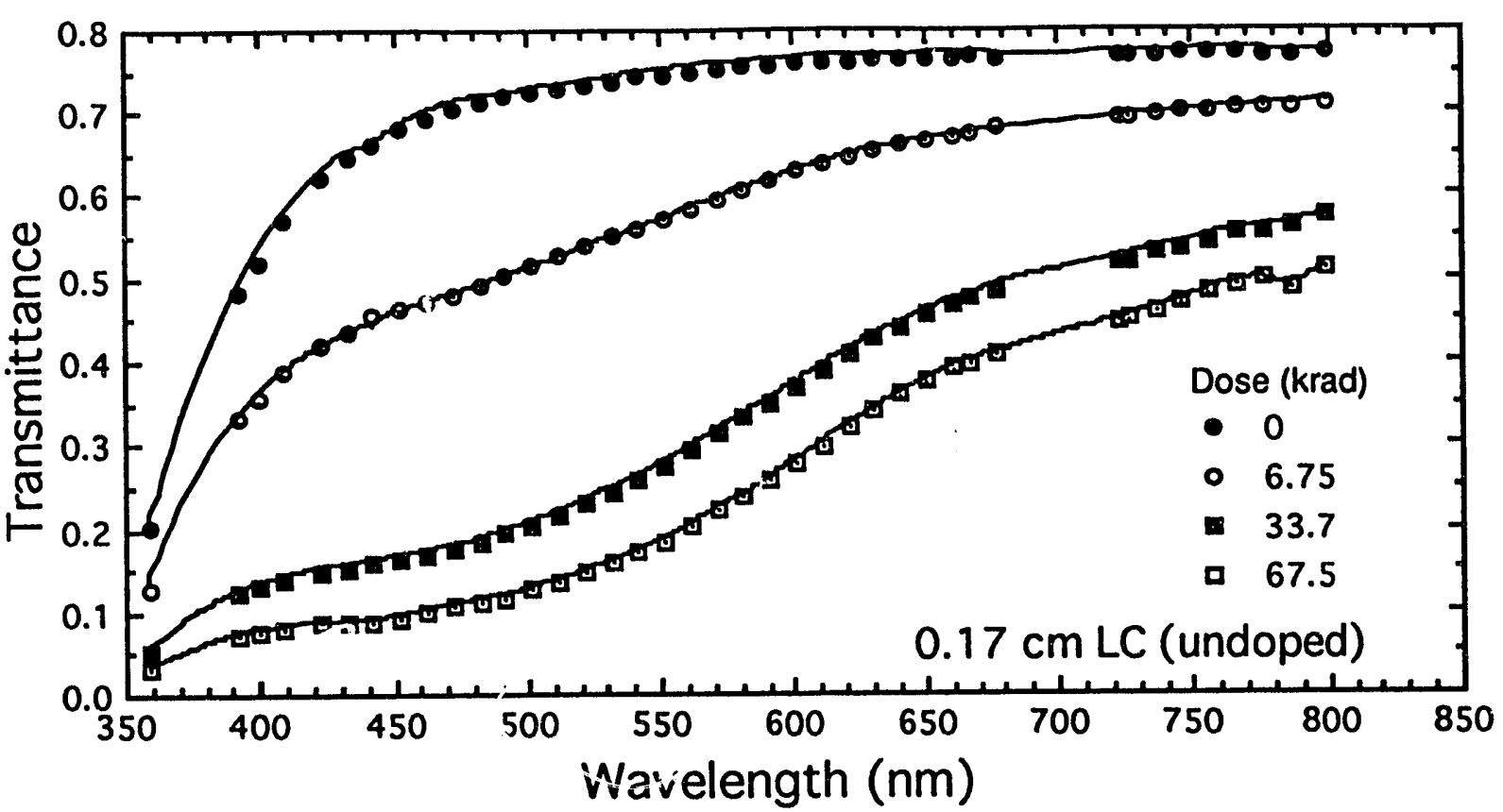

Fig. 4a: Transmittance vs. wavelength for $0.17 \mathrm{~cm}$ of LC fiber optics (Gallileo Electro-Optic) after exposure to various radiation doses. The plotted points are measured data; the solid lines are the fits using the "best fit" values to the radiation induced absorption parameters appearing in Eqs. 1 and 2. (The "best fit" values are shown as dots in the plots below.)
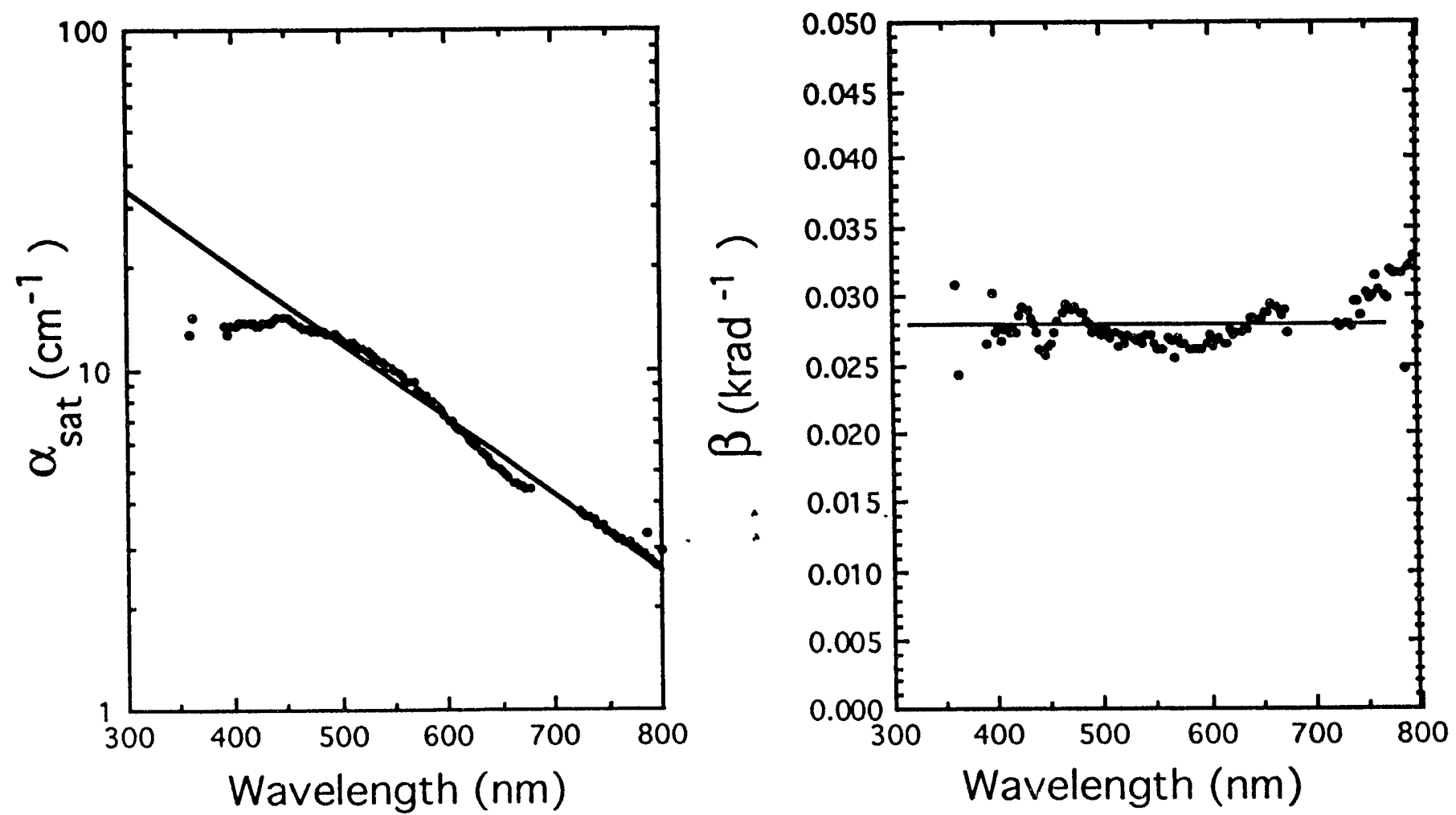

Fig. 4b: Radiation Induced Absorption Parameters vs. Wavelength for undoped LC fiber optics. Dots are values given by least squares fits, solid lines are suggested analytic approximations to the fitted data. 
Schott Fiber Optic RWY 47 (undoped)

Table 4a: Least Squares Fit to $T_{0}, \alpha_{\text {sat }}$ and $\beta$; Schott Fiber Optic RWY 47.

\begin{tabular}{|c|c|c|c|c|}
\hline$\lambda(\mathrm{nm})$ & $\mathrm{T}_{0}$ & $\alpha_{\text {sat }}\left(\mathrm{cm}^{-1}\right)$ & $\beta\left(\mathrm{krad}^{-1}\right)$ & error $(\mathrm{rms} \%)$ \\
\hline 362.79 & $2.39 \mathrm{E}-02$ & $1.77 \mathrm{E}+00$ & $3.00 \mathrm{E}-02$ & $10.51 \%$ \\
399.14 & $2.40 \mathrm{E}-01$ & $5.25 \mathrm{E}+00$ & $5.17 \mathrm{E}-03$ & $0.68 \%$ \\
422.27 & $3.70 \mathrm{E}-01$ & $3.17 \mathrm{E}+00$ & $8.99 \mathrm{E}-03$ & $0.73 \%$ \\
461.93 & $4.89 \mathrm{E}-01$ & $2.32 \mathrm{E}+00$ & $1.11 \mathrm{E}-02$ & $0.86 \%$ \\
501.58 & $5.55 \mathrm{E}-01$ & $2.47 \mathrm{E}+00$ & $7.71 \mathrm{E}-03$ & $0.67 \%$ \\
541.24 & $6.05 \mathrm{E}-01$ & $1.83 \mathrm{E}+00$ & $8.49 \mathrm{E}-03$ & $0.42 \%$ \\
580.89 & $6.41 \mathrm{E}-01$ & $1.49 \mathrm{E}+00$ & $8.39 \mathrm{E}-03$ & $0.50 \%$ \\
620.55 & $6.67 \mathrm{E}-01$ & $1.20 \mathrm{E}+00$ & $8.40 \mathrm{E}-03$ & $0.43 \%$ \\
640.37 & $6.76 \mathrm{E}-01$ & $9.99 \mathrm{E}-01$ & $9.17 \mathrm{E}-03$ & $0.46 \%$ \\
680.03 & $6.85 \mathrm{E}-01$ & $1.69 \mathrm{E}+00$ & $3.69 \mathrm{E}-03$ & $0.87 \%$ \\
722.99 & $6.90 \mathrm{E}-01$ & $1.34 \mathrm{E}+00$ & $3.51 \mathrm{E}-03$ & $0.60 \%$ \\
762.64 & $6.92 \mathrm{E}-01$ & $5.09 \mathrm{E}-01$ & $8.73 \mathrm{E}-03$ & $0.36 \%$ \\
798.99 & $6.81 \mathrm{E}-01$ & $4.27 \mathrm{E}-01$ & $8.95 \mathrm{E}-03$ & $0.22 \%$ \\
815.52 & $6.79 \mathrm{E}-01$ & $3.17 \mathrm{E}-01$ & $1.29 \mathrm{E}-02$ & $0.25 \%$ \\
\hline
\end{tabular}

Table 5b: Analytic Approximations for $\alpha_{\text {sat }}$ and $\beta$, Undoped RWY 47 Fiber Optics $\alpha_{\text {sat }}=30.860 \exp (-.0051430 \lambda) \mathrm{cm}^{-1}, \beta=.0085 \mathrm{krad}^{-1}$

\begin{tabular}{|c|c|c|c|c|}
\hline$\lambda(\mathrm{nm})$ & $\mathrm{T}_{0}$ & $\alpha_{\text {sat }}(\mathrm{cm}-1)$ & $\beta\left(\mathrm{krad}^{-1}\right)$ & error $(\mathrm{rms} \%)$ \\
\hline 362.79 & $2.39 \mathrm{E}-02$ & $4.78 \mathrm{E}+00$ & $8.50 \mathrm{E}-03$ & $18.82 \%$ \\
399.14 & $2.40 \mathrm{E}-01$ & $3.96 \mathrm{E}+00$ & $8.50 \mathrm{E}-03$ & $6.86 \%$ \\
422.27 & $3.70 \mathrm{E}-01$ & $3.52 \mathrm{E}+00$ & $8.50 \mathrm{E}-03$ & $3.06 \%$ \\
461.93 & $4.89 \mathrm{E}-01$ & $2.87 \mathrm{E}+00$ & $8.50 \mathrm{E}-03$ & $1.26 \%$ \\
501.58 & $5.55 \mathrm{E}-01$ & $2.34 \mathrm{E}+00$ & $8.50 \mathrm{E}-03$ & $1.06 \%$ \\
541.24 & $6.05 \mathrm{E}-01$ & $1.91 \mathrm{E}+00$ & $8.50 \mathrm{E}-03$ & $1.21 \%$ \\
580.89 & $6.41 \mathrm{E}-01$ & $1.56 \mathrm{E}+00$ & $8.50 \mathrm{E}-03$ & $1.22 \%$ \\
620.55 & $6.67 \mathrm{E}-01$ & $1.27 \mathrm{E}+00$ & $8.50 \mathrm{E}-03$ & $1.25 \%$ \\
640.37 & $6.76 \mathrm{E}-01$ & $1.15 \mathrm{E}+00$ & $8.50 \mathrm{E}-03$ & $1.35 \%$ \\
680.03 & $6.85 \mathrm{E}-01$ & $9.35 \mathrm{E}-01$ & $8.50 \mathrm{E}-03$ & $1.72 \%$ \\
722.99 & $6.90 \mathrm{E}-01$ & $7.49 \mathrm{E}-01$ & $8.50 \mathrm{E}-03$ & $1.78 \%$ \\
762.64 & $6.92 \mathrm{E}-01$ & $6.11 \mathrm{E}-01$ & $8.50 \mathrm{E}-03$ & $1.37 \%$ \\
798.99 & $6.81 \mathrm{E}-01$ & $5.07 \mathrm{E}-01$ & $8.50 \mathrm{E}-03$ & $0.93 \%$ \\
815.52 & $6.79 \mathrm{E}-01$ & $4.66 \mathrm{E}-01$ & $8.50 \mathrm{E}-03$ & $0.59 \%$ \\
\hline
\end{tabular}




\section{Schott Fiber Optic RWY 47 (undoped)}

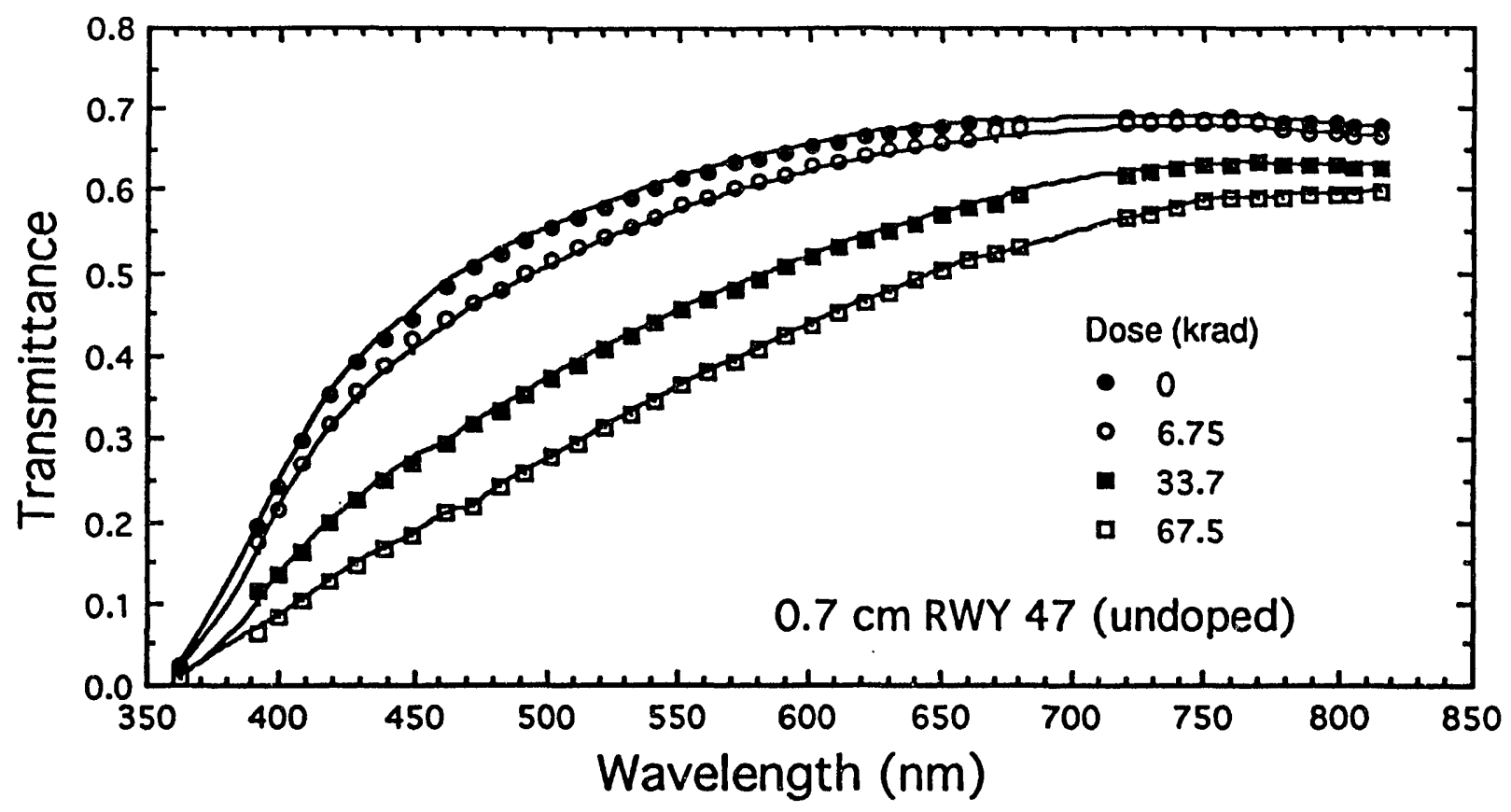

Fig. 5a: Transmittance vs. wavelength for $0.7 \mathrm{~cm}$ of RWY 47 fiber optics (Schot Fiber Optic) after exposure to various radiation doses. The plotted points are measured data; the solid lines are the fits using the "best fit" values to the radiation induced absorption parameters appearing in Eqs. 1 and 2. (The "best fit" values are shown as dots in the plots below.)
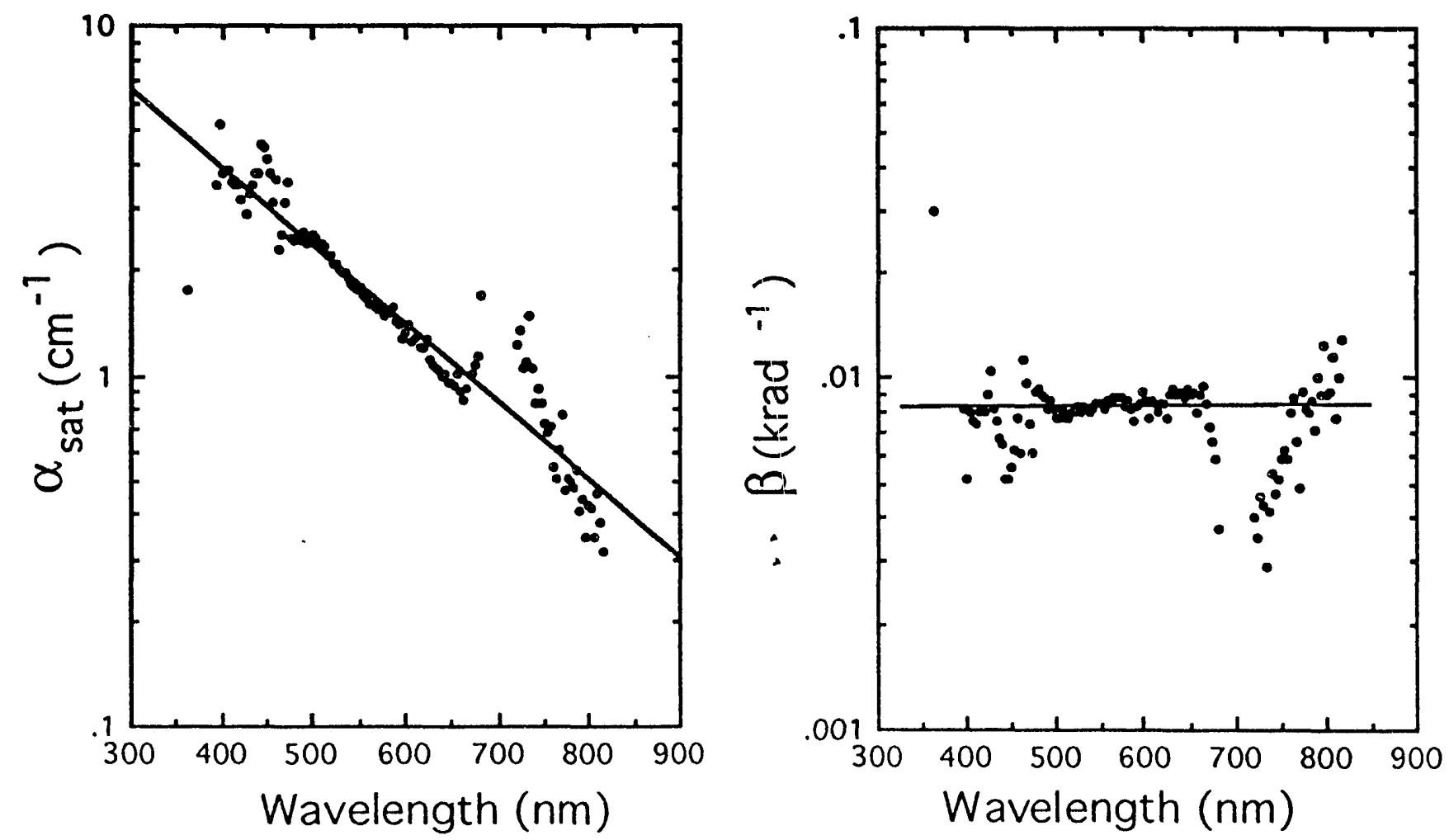

Fig. 5b: Radiation Induced Absorption Parameters vs. Wavelength for Schotl Fiber Optic undoped RWY 47. Dots are values given by least squares fits, solid lines are suggested analytic approximations to the fitted data. 


\section{Schott LaK 9 G15}

Table 6a: Least Squares Fit to $T_{0}, \alpha_{\text {sat }}$ and $\beta$; Schott LaK 9 G15.

(Bold faced values of $\alpha_{\text {sat }}$ and $\beta$ are extrapolations based on Table $6 \mathrm{~b}$ )

\begin{tabular}{|c|c|c|c|c|}
\hline$\lambda(\mathrm{nm})$ & $\mathrm{T}_{0}$ & $\alpha_{\text {sat }}\left(\mathrm{cm}^{-1}\right)$ & $\beta\left(\mathrm{krad}^{-1}\right)$ & error (rms\%) \\
\hline 360 & $2.00 \mathrm{E}-01$ & insufficient data & insufficient data & insufficient data \\
400 & $5.10 \mathrm{E}-01$ & insufficient data & insufficient data & insufficient data \\
440 & $7.05 \mathrm{E}-01$ & $1.51 \mathrm{E}+00$ & $1.90 \mathrm{E}-03$ & $5.06 \%$ \\
480 & $8.27 \mathrm{E}-01$ & $4.96 \mathrm{E}-01$ & $1.95 \mathrm{E}-03$ & $1.15 \%$ \\
520 & $8.52 \mathrm{E}-01$ & $2.42 \mathrm{E}-01$ & $1.84 \mathrm{E}-03$ & $0.51 \%$ \\
560 & $8.59 \mathrm{E}-01$ & $1.47 \mathrm{E}-01$ & $1.78 \mathrm{E}-03$ & $0.45 \%$ \\
600 & $8.64 \mathrm{E}-01$ & $9.58 \mathrm{E}-02$ & $1.88 \mathrm{E}-03$ & $0.36 \%$ \\
680 & $8.73 \mathrm{E}-01$ & $4.65 \mathrm{E}-02$ & $2.10 \mathrm{E}-03$ & $0.22 \%$ \\
760 & $8.76 \mathrm{E}-01$ & $2.50 \mathrm{E}-02$ & $1.78 \mathrm{E}-03$ & $0.21 \%$ \\
840 & $8.79 \mathrm{E}-01$ & $1.55 \mathrm{E}-02$ & $1.90 \mathrm{E}-03$ & $0.17 \%$ \\
920 & $8.79 \mathrm{E}-01$ & $1.04 \mathrm{E}-02$ & $1.90 \mathrm{E}-03$ & $0.15 \%$ \\
1000 & $8.79 \mathrm{E}-01$ & $\mathbf{7 . 4 8 E}-03$ & $1.90 \mathrm{E}-03$ & $0.13 \%$ \\
1100 & $8.79 \mathrm{E}-01$ & $5.29 \mathrm{E}-03$ & $1.90 \mathrm{E}-03$ & $0.15 \%$ \\
1200 & $8.79 \mathrm{E}-01$ & $3.96 \mathrm{E}-03$ & $1.90 \mathrm{E}-03$ & $0.30 \%$ \\
\hline
\end{tabular}

Table 6b: Analytic Approximations for $\alpha_{\text {sat }}$ and $\beta$, Schott LaK 9 G15

\begin{tabular}{|c|c|c|c|c|}
\hline \multicolumn{5}{|c|}{$\alpha_{\text {sat }}=1.6357 \times 10^{-4} \exp (-3823 / \lambda) \mathrm{cm}^{-1}, \beta=.0019 \mathrm{krad}^{-1}$} \\
\hline$\lambda(\mathrm{nm})$ & $\mathrm{T}_{0}$ & $\alpha_{\text {sat }}\left(\mathrm{cm}^{-1}\right)$ & $\beta\left(\mathrm{krad}^{-1}\right)$ & error $(\mathrm{rms} \%)$ \\
\hline 380 & $2.00 \mathrm{E}-01$ & $3.83 \mathrm{E}+00$ & $1.90 \mathrm{E}-03$ & insufficient data \\
400 & $5.10 \mathrm{E}-01$ & $2.32 \mathrm{E}+00$ & $1.90 \mathrm{E}-03$ & insufficient data \\
440 & $7.05 \mathrm{E}-01$ & $9.71 \mathrm{E}-01$ & $1.90 \mathrm{E}-03$ & $35.00 \%$ \\
480 & $8.27 \mathrm{E}-01$ & $4.71 \mathrm{E}-01$ & $1.90 \mathrm{E}-03$ & $2.18 \%$ \\
520 & $8.52 \mathrm{E}-01$ & $2.55 \mathrm{E}-01$ & $1.90 \mathrm{E}-03$ & $1.10 \%$ \\
560 & $8.59 \mathrm{E}-01$ & $1.51 \mathrm{E}-01$ & $1.90 \mathrm{E}-03$ & $0.63 \%$ \\
600 & $8.64 \mathrm{E}-01$ & $9.57 \mathrm{E}-02$ & $1.90 \mathrm{E}-03$ & $0.36 \%$ \\
680 & $8.73 \mathrm{E}-01$ & $4.52 \mathrm{E}-02$ & $1.90 \mathrm{E}-03$ & $0.27 \%$ \\
760 & $8.76 \mathrm{E}-01$ & $2.50 \mathrm{E}-02$ & $1.90 \mathrm{E}-03$ & $0.21 \%$ \\
840 & $8.79 \mathrm{E}-01$ & $1.55 \mathrm{E}-02$ & $1.90 \mathrm{E}-03$ & $0.17 \%$ \\
920 & $8.79 \mathrm{E}-01$ & $1.04 \mathrm{E}-02$ & $1.90 \mathrm{E}-03$ & $0.15 \%$ \\
1000 & $8.79 \mathrm{E}-01$ & $7.48 \mathrm{E}-03$ & $1.90 \mathrm{E}-03$ & $0.13 \%$ \\
1100 & $8.79 \mathrm{E}-01$ & $5.29 \mathrm{E}-03$ & $1.90 \mathrm{E}-03$ & $0.15 \%$ \\
1200 & $8.79 \mathrm{E}-01$ & $3.96 \mathrm{E}-03$ & $1.90 \mathrm{E}-03$ & $0.30 \%$ \\
\hline
\end{tabular}


Schott LaK 9 G15

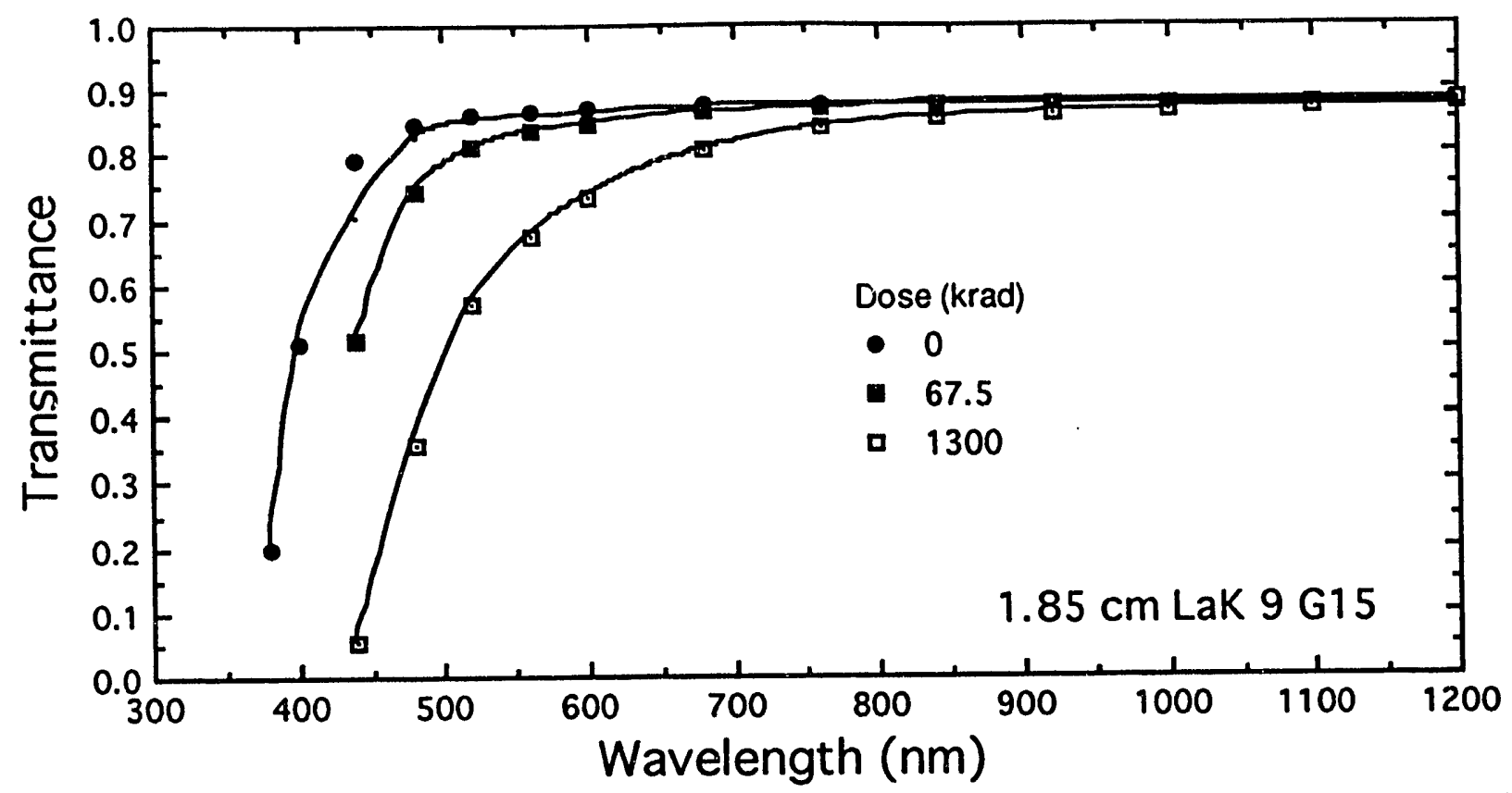

Fig, 6a: Transmittance vs. wavelength for $1.85 \mathrm{~cm}$ of Schott LaK $9 \mathrm{G} 15$ after exposure to various radiation doses. The plotted points are measured data; the solid lines are the fits using the "best fit" values to the radiation induced absorption parameters appearing in Eqs. 1 and 2. (The "best fit" values are shown as dots in the plots below.)
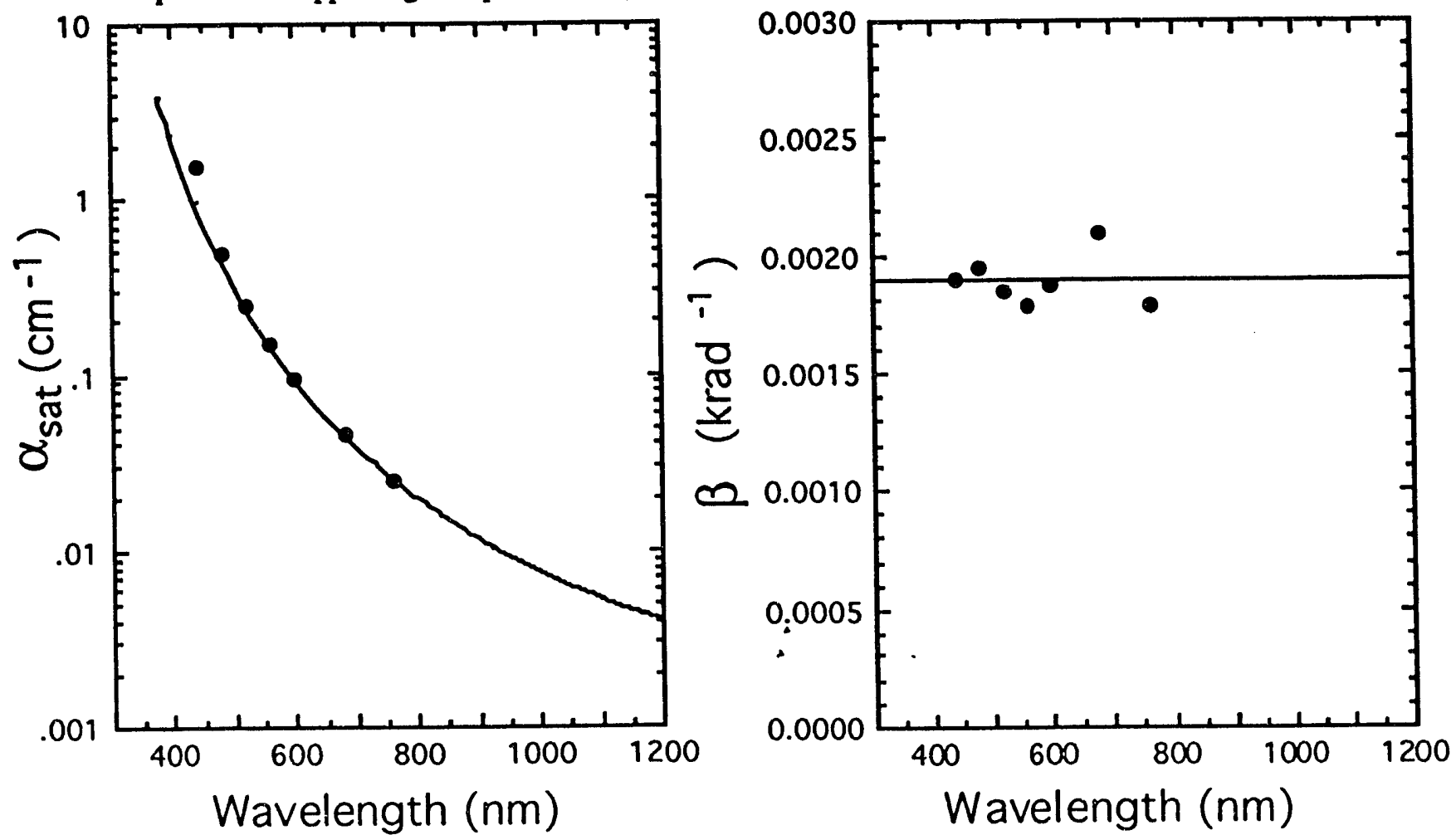

Fig. 6b: Radiation Induced Absorption Parameters vs. Wavelength for Schott LaK 9 G15. Dots are values given by least squares fits, solid lines are suggested analytic approximations to the fitted data. 
Schott SF 6 G5

Table 7a: Least Squares Fit to $T_{0}, \alpha_{\text {sat }}$ and $\beta$, Schott SF 6 G5

\begin{tabular}{|c|c|c|c|c|}
\hline$\lambda(\mathrm{nm})$ & $\mathrm{T}_{0}$ & $\alpha_{\text {sat }}\left(\mathrm{cm}^{-1}\right)$ & $\beta\left(\mathrm{krad}^{-1}\right)$ & error $(\mathrm{rms} \%)$ \\
\hline 380 & \multicolumn{4}{|c|}{ too opaque to measure } \\
400 & \multicolumn{4}{|c|}{ too opaque to measure } \\
440 & $1.30 \mathrm{E}-02$ & $9.92 \mathrm{E}-01$ & $6.55 \mathrm{E}+00$ & $6.45 \%$ \\
480 & $3.95 \mathrm{E}-01$ & $8.29 \mathrm{E}-01$ & $3.94 \mathrm{E}-03$ & $2.69 \%$ \\
520 & $7.31 \mathrm{E}-01$ & $2.34 \mathrm{E}-01$ & $3.59 \mathrm{E}-03$ & $0.51 \%$ \\
560 & $8.13 \mathrm{E}-01$ & $7.85 \mathrm{E}-02$ & $2.72 \mathrm{E}-03$ & $0.20 \%$ \\
600 & $8.32 \mathrm{E}-01$ & $3.64 \mathrm{E}-02$ & $2.56 \mathrm{E}-03$ & $0.18 \%$ \\
680 & $8.43 \mathrm{E}-01$ & $1.90 \mathrm{E}-02$ & $2.70 \mathrm{E}-03$ & $0.18 \%$ \\
760 & $8.48 \mathrm{E}-01$ & $1.57 \mathrm{E}-02$ & $4.86 \mathrm{E}-03$ & $0.15 \%$ \\
840 & $8.50 \mathrm{E}-01$ & $1.57 \mathrm{E}-02$ & $1.12 \mathrm{E}-02$ & $0.28 \%$ \\
920 & $8.51 \mathrm{E}-01$ & $1.47 \mathrm{E}-02$ & $1.19 \mathrm{E}-02$ & $0.27 \%$ \\
1000 & $8.58 \mathrm{E}-01$ & $1.28 \mathrm{E}-02$ & $2.34 \mathrm{E}+00$ & $0.17 \%$ \\
1100 & $8.59 \mathrm{E}-01$ & $1.16 \mathrm{E}-02$ & $1.97 \mathrm{E}+00$ & $0.07 \%$ \\
1200 & $8.60 \mathrm{E}-01$ & $1.16 \mathrm{E}-02$ & $1.97 \mathrm{E}+00$ & $0.07 \%$ \\
\hline
\end{tabular}

Table 6b: Analytic Approximations for $\alpha_{\text {sat }}$ and $\beta$, Schott SF 6 G5 $\lambda<630 \mathrm{~nm}: \alpha_{\text {sat }}=2.5758 \times 10^{4} \exp \left(-2.2419 \times 10^{-2} \lambda\right) \mathrm{cm}^{-1}, \beta=.006 \mathrm{krad}^{-1}$ $\lambda>630 \mathrm{~nm}: \alpha_{\text {sat }}=3.4322 \times 10^{-2} \exp \left(-9.4761 \times 10^{-4} \lambda\right) \mathrm{cm}^{-1}, \beta=.006 \mathrm{krad}^{-1}$

\begin{tabular}{|c|c|c|c|c|}
\hline$\lambda(\mathrm{nm})$ & $\mathrm{T}_{0}$ & $\alpha_{\text {sat }}\left(\mathrm{cm}^{-1}\right)$ & $\beta\left(\mathrm{krad}^{-1}\right)$ & error $(\mathrm{rms} \%)$ \\
\hline 380 & \multicolumn{4}{|c|}{ too opaque to measure } \\
400 & \multicolumn{4}{|c|}{ too opaque to measure } \\
440 & $1.30 \mathrm{E}-02$ & $1.34 \mathrm{E}+00$ & $6.00 \mathrm{E}-03$ & $65.97 \%$ \\
480 & $3.95 \mathrm{E}-01$ & $5.46 \mathrm{E}-01$ & $6.00 \mathrm{E}-03$ & $9.14 \%$ \\
520 & $7.31 \mathrm{E}-01$ & $2.23 \mathrm{E}-01$ & $6.00 \mathrm{E}-03$ & $0.87 \%$ \\
560 & $8.13 \mathrm{E}-01$ & $9.09 \mathrm{E}-02$ & $6.00 \mathrm{E}-03$ & $0.68 \%$ \\
600 & $8.32 \mathrm{E}-01$ & $3.71 \mathrm{E}-02$ & $6.00 \mathrm{E}-03$ & $0.26 \%$ \\
680 & $8.43 \mathrm{E}-01$ & $1.79 \mathrm{E}-02$ & $6.00 \mathrm{E}-03$ & $0.19 \%$ \\
760 & $8.48 \mathrm{E}-01$ & $1.66 \mathrm{E}-02$ & $6.00 \mathrm{E}-03$ & $0.16 \%$ \\
840 & $8.50 \mathrm{E}-01$ & $1.54 \mathrm{E}-02$ & $6.00 \mathrm{E}-03$ & $0.29 \%$ \\
920 & $8.51 \mathrm{E}-01$ & $1.42 \mathrm{E}-02$ & $6.00 \mathrm{E}-03$ & $0.29 \%$ \\
1000 & $8.58 \mathrm{E}-01$ & $1.32 \mathrm{E}-02$ & $6.00 \mathrm{E}-03$ & $0.71 \%$ \\
1100 & $8.59 \mathrm{E}-01$ & $1.20 \mathrm{E}-02$ & $6.00 \mathrm{E}-03$ & $0.67 \%$ \\
1200 & $8.60 \mathrm{E}-01$ & $1.09 \mathrm{E}-02$ & $6.00 \mathrm{E}-03$ & $0.68 \%$ \\
\hline
\end{tabular}


Schott SF 6 G5

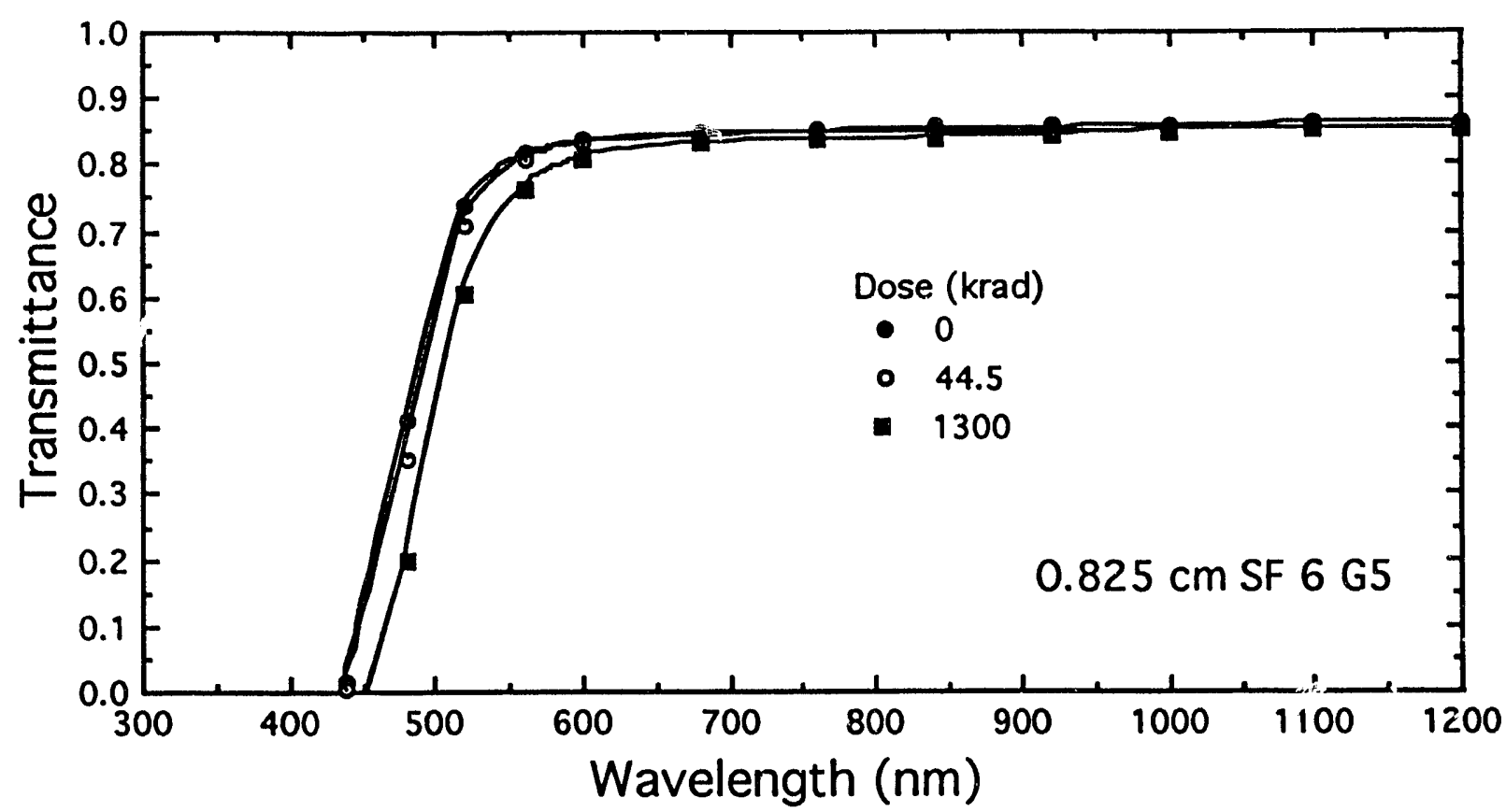

Fig. 7a: Transmittance vs. wavelength for $0.825 \mathrm{~cm}$ of Schott SF 6 G5 after exposure to various radiation doses. The plotted points are measured data; the solid lines are the fits using the "best fit" values to the radiation inciuced absorption parameters appearing in Eqs. 1 and 2. (The "best fit" values are shown as dots in the plots below.)
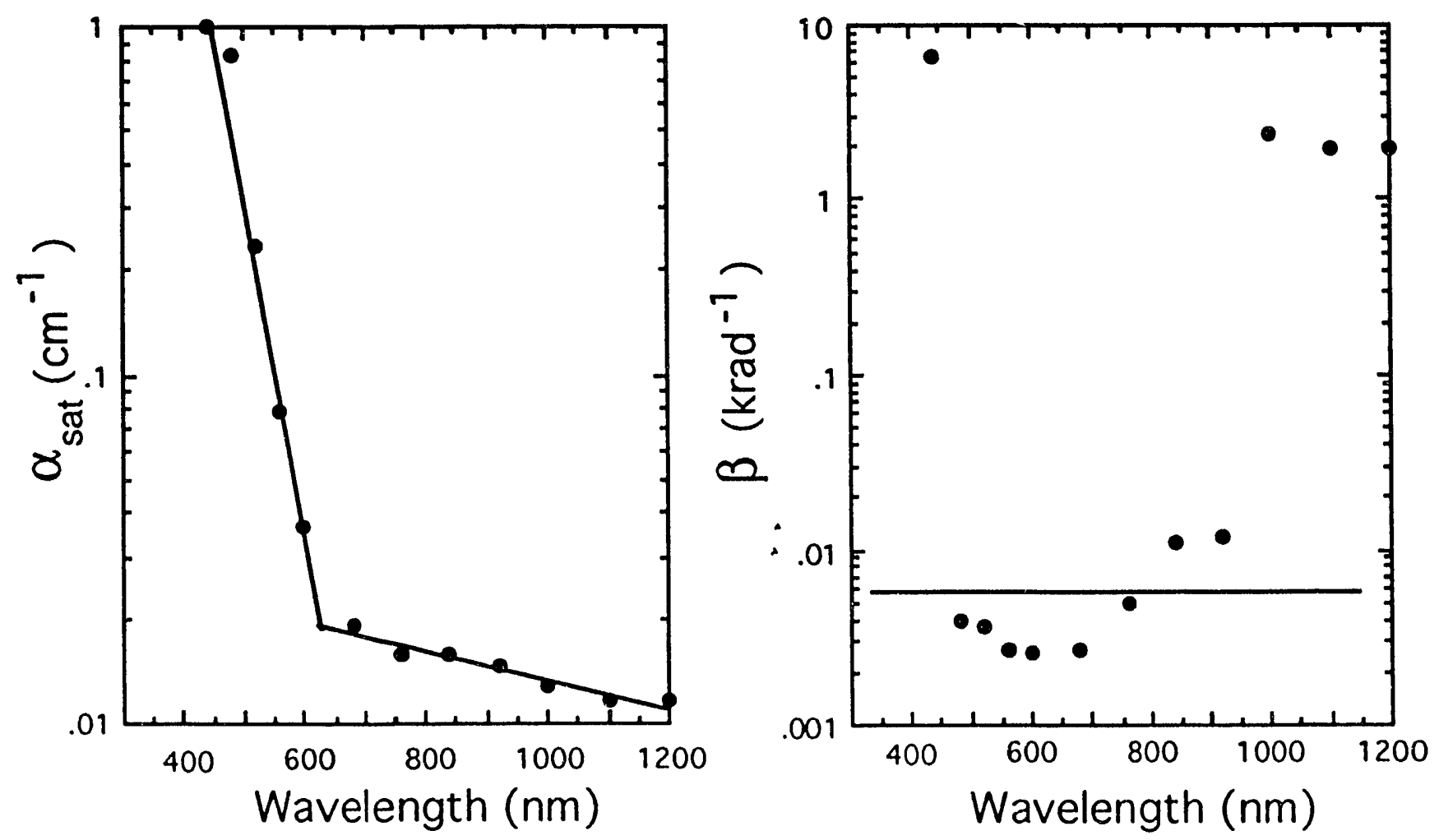

Fig. 7b: Radiation Induced Absorption Parameters vs. Wavelength for Schott SF 6 G5. Dots are values given by least squares fits, solid lines are suggested analytic approximations to the fitted data. 


\section{Gallileo Electro-Optic Ce Doped LC Fiber Optics}

Table 8a: Least Squares Fit to $T_{0}, \alpha_{\text {sat }}$ and $\beta$; Ce doped LC fiber optics.

\begin{tabular}{|c|c|c|c|c|}
\hline$\lambda(\mathrm{nm})$ & $\mathrm{T}_{0}$ & $\alpha_{\text {sat }}\left(\mathrm{cm}^{-1}\right)$ & $\beta\left(\mathrm{krad}^{-1}\right)$ & error (rms\%) \\
\hline 432 & $3.65 \mathrm{E}-02$ & $2.41 \mathrm{E}+00$ & $1.41 \mathrm{E}-01$ & $4.55 \%$ \\
442 & $1.11 \mathrm{E}-01$ & $2.03 \mathrm{E}+00$ & $1.52 \mathrm{E}-01$ & $4.34 \%$ \\
452 & $2.18 \mathrm{E}-01$ & $2.05 \mathrm{E}+00$ & $3.75 \mathrm{E}-02$ & $5.18 \%$ \\
462 & $3.70 \mathrm{E}-01$ & $8.47 \mathrm{E}+02$ & $2.88 \mathrm{E}-05$ & $3.06 \%$ \\
475 & $4.91 \mathrm{E}-01$ & $1.49 \mathrm{E}+00$ & $1.06 \mathrm{E}-02$ & $1.61 \%$ \\
502 & $6.72 \mathrm{E}-01$ & $2.06 \mathrm{E}+02$ & $3.29 \mathrm{E}-05$ & $0.82 \%$ \\
538 & $7.57 \mathrm{E}-01$ & $1.79 \mathrm{E}+02$ & $2.30 \mathrm{E}-05$ & $0.47 \%$ \\
581 & $7.62 \mathrm{E}-01$ & $1.56 \mathrm{E}+02$ & $1.26 \mathrm{E}-05$ & $0.53 \%$ \\
601 & $7.77 \mathrm{E}-01$ & $1.35 \mathrm{E}+02$ & $1.57 \mathrm{E}-05$ & $0.50 \%$ \\
621 & $7.85 \mathrm{E}-01$ & $1.14 \mathrm{E}+02$ & $1.36 \mathrm{E}-05$ & $0.51 \%$ \\
640 & $7.85 \mathrm{E}-01$ & $9.46 \mathrm{E}+01$ & $1.22 \mathrm{E}-05$ & $0.49 \%$ \\
667 & $7.85 \mathrm{E}-01$ & $7.67 \mathrm{E}+01$ & $1.47 \mathrm{E}-05$ & $0.50 \%$ \\
723 & $7.80 \mathrm{E}-01$ & $1.09 \mathrm{E}+02$ & $1.54 \mathrm{E}-05$ & $0.51 \%$ \\
746 & $7.66 \mathrm{E}-01$ & $1.33 \mathrm{E}+02$ & $8.73 \mathrm{E}-06$ & $0.53 \%$ \\
763 & $7.73 \mathrm{E}-01$ & $8.48 \mathrm{E}+01$ & $1.34 \mathrm{E}-05$ & $0.56 \%$ \\
799 & $7.51 \mathrm{E}-01$ & $1.70 \mathrm{E}+01$ & $1.27 \mathrm{E}-05$ & $0.47 \%$ \\
\hline
\end{tabular}

Radiation induced absorption for the Ce doped LC fiber optics is very small. Consequently the values of $\alpha_{\text {sat }}$ determined by fits to the data show a noisy and erratic dependence on wavelength. The "best fit" values of $\alpha_{\text {sat }}$ are probably highly inaccurate. Therefore, no simple analytic approximation to $\alpha_{\text {sat }}$ was attempted. Though the best fit values of $\alpha_{\text {sat }}$ tend to be large, the values of $\beta$ are very small. For $500 \mathrm{~nm}<\lambda<800 \mathrm{~nm}, \beta \sim 1 \times 10^{-5}$ to $2 \times 10^{-5} \mathrm{krad}^{-1}$ and hence the onset of saturation does not occur until doses approach 50 to $100 \mathrm{Mrad}$. 


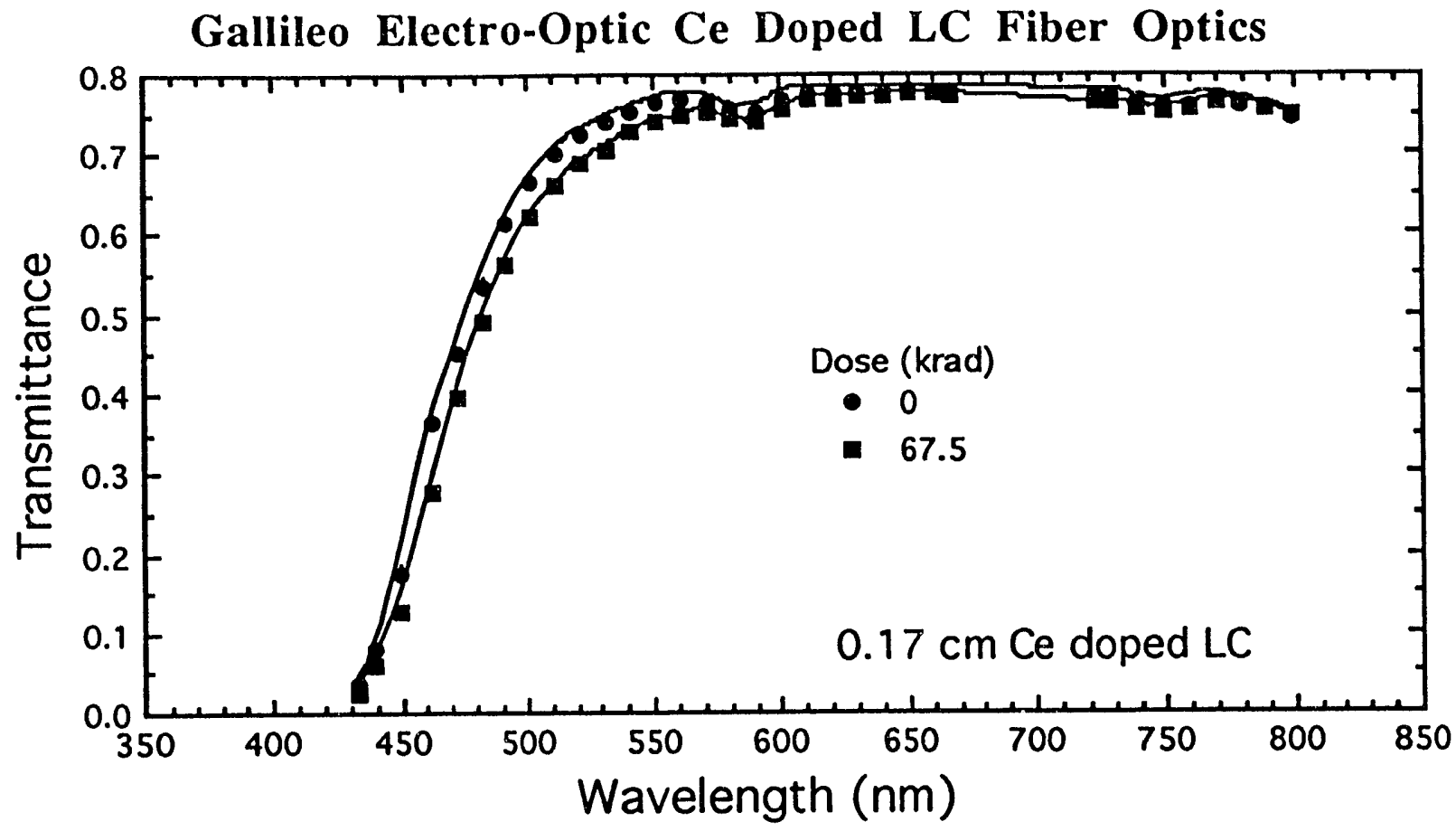

Fig. 8a: Transmittance vs. wavelength for $0.17 \mathrm{~cm}$ of Ce doped LC fiber optics (Gallileo Electro-Optic) after exposure to various radiation doses. The plotted points are measured data; the solid lines are the fits using the "best fit" values to the radiation induced absorption parameters appearing in Eqs. 1 and 2. (The "best fit" values are shown as dots in the plots below.)
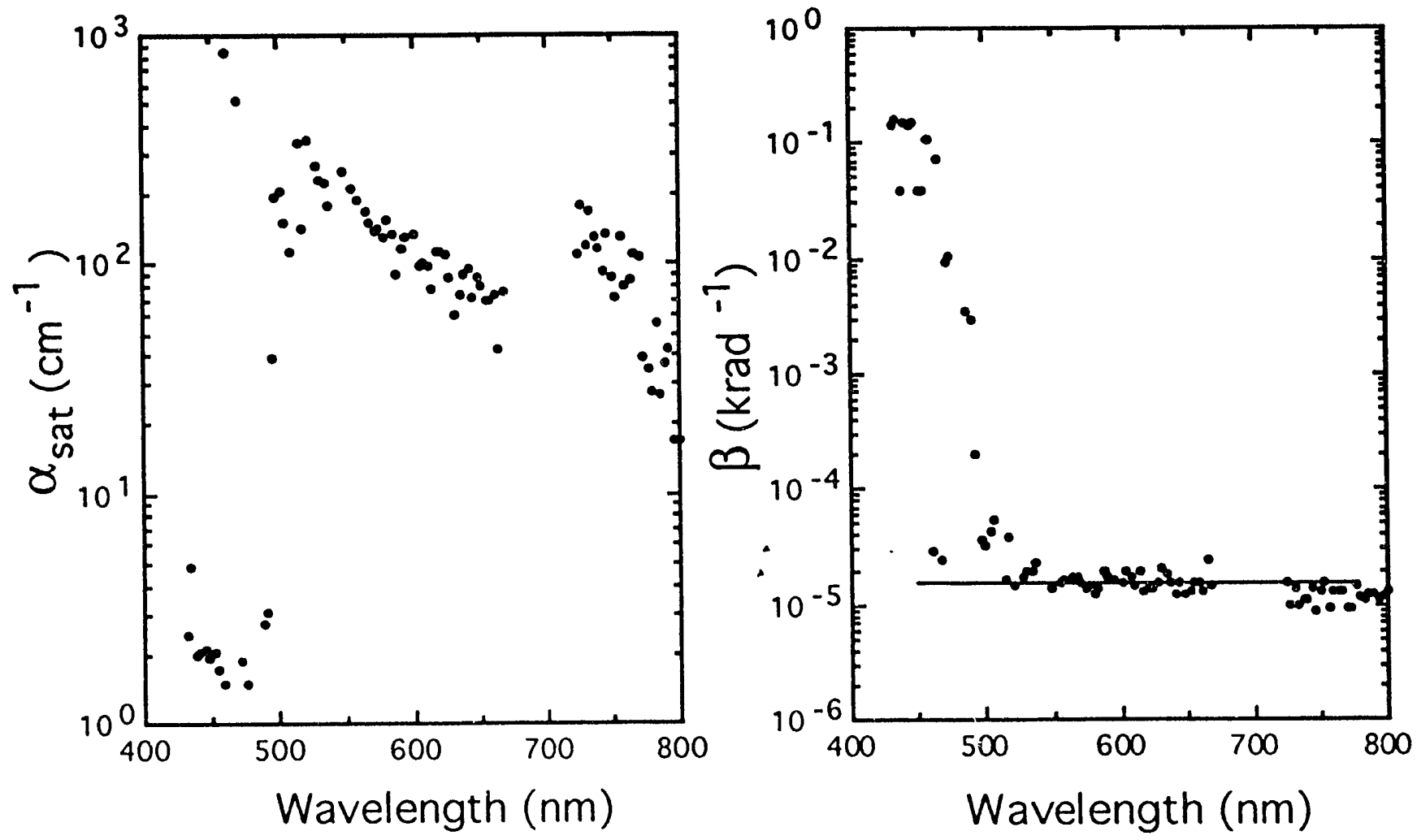

Fig. 4b: Radiation Induced Absorption Parameters vs. Wavelength for Ce doped LC fiber optics. Dots are values given by least squares fits, solid lines are suggested analytic approximations to the fitted data. No analytic approximation to $\alpha_{\text {sat }}$ was attempted. 
Schott Fiber Optic RWY 47 (Ce Doped)

Table 9a: Least Squares Fit to To, $\alpha_{\text {sat }}$ and $\beta$; Ce Doped RWY 47.

Bold faced values are extrapolations using the analytic fits of table $9 \mathrm{~b}$.

\begin{tabular}{|c|c|c|c|c|}
\hline$\lambda(\mathrm{nm})$ & $T_{0}$ & $\alpha_{\text {sat }}\left(\mathrm{cm}^{-1}\right)$ & $\beta\left(\mathrm{krad}^{-1}\right)$ & error (rms\%) \\
\hline 435 & $9.04 \mathrm{E}-03$ & $1.89 \mathrm{E}+00$ & $1.37 \mathrm{E}-01$ & $18.59 \%$ \\
\hline 445 & 2.43E-02 & $1.02 E+00$ & $1.09 \mathrm{E}-01$ & $15.68 \%$ \\
\hline 455 & 6.82E-02 & 6.32E-01 & $9.15 \mathrm{E}-02$ & $6.97 \%$ \\
\hline 465 & $1.24 \mathrm{E}-01$ & $5.20 \mathrm{E}-01$ & 8.11E-02 & $5.95 \%$ \\
\hline 485 & 2.71E-01 & 2.64E-01 & $6.83 \mathrm{E}-02$ & $2.39 \%$ \\
\hline 502 & $3.73 j-01$ & $1.45 ;-01$ & 5.00 E-02 & $1.91 \%$ \\
\hline 521 & 4.77E-01 & $9.42 \mathrm{i} \cdot 02$ & $5.83 \mathrm{E}-02$ & $0.80 \%$ \\
\hline 541 & $5.41 \mathrm{E}-01$ & $4.94 \mathrm{E}-02$ & 4.54E-02 & $0.28 \%$ \\
\hline 554 & 5.73E-01 & $3.65 \mathrm{E}-02$ & 4.03E-02 & $0.33 \%$ \\
\hline 601 & $6.35 \mathrm{E}-01$ & $7.24 E-03$ & $1.58 \mathrm{E}-02$ & $0.61 \%$ \\
\hline 650 & $6.66 \mathrm{E}-01$ & $1.47 \mathrm{E}-03$ & $0.00 E+00$ & $0.54 \%$ \\
\hline 723 & $6.89 \mathrm{E}-01$ & $1.41 \mathrm{E}-04$ & $0.00 E+00$ & $0.80 \%$ \\
\hline 782 & $6.81 \mathrm{E}-01$ & 2.07E-05 & $0.00 E+00$ & $0.56 \%$ \\
\hline 816 & $6.16 \mathrm{E}-01$ & $7.15 \mathrm{E}-06$ & $0.00 \mathrm{E}+00$ & $0.23 \%$ \\
\hline
\end{tabular}

Table 9b: Analytic Approximations for $\alpha_{\text {sat }}$ and $\beta$, Ce Doped RWY 47. $\alpha_{\text {sat }}=1.8389 \times 10^{6} \exp (-.032215 \lambda) \mathrm{cm}^{-1}, \beta=\min \left(0, .33740-5.3538 \times 10^{-4} \lambda\right) \mathrm{krad}^{-1}$

\begin{tabular}{|c|c|c|c|c|}
\hline$\lambda(\mathrm{nm})$ & $\mathrm{T}_{0}$ & $\alpha_{\text {sat }}\left(\mathrm{cm}^{-1}\right)$ & $\beta\left(\mathrm{krad}^{-1}\right)$ & error $(\mathrm{rms} \%)$ \\
\hline 435 & $9.04 \mathrm{E}-03$ & $1.48 \mathrm{E}+00$ & $1.04 \mathrm{E}-01$ & $28.74 \%$ \\
445 & $2.43 \mathrm{E}-02$ & $1.08 \mathrm{E}+00$ & $9.89 \mathrm{E}-02$ & $15.80 \%$ \\
455 & $6.82 \mathrm{E}-02$ & $7.84 \mathrm{E}-01$ & $9.36 \mathrm{E}-02$ & $9.56 \%$ \\
465 & $1.24 \mathrm{E}-01$ & $5.70 \mathrm{E}-01$ & $8.83 \mathrm{E}-02$ & $6.43 \%$ \\
485 & $2.71 \mathrm{E}-01$ & $3.0 \mathrm{E}-01$ & $7.77 \mathrm{E}-02$ & $2.99 \%$ \\
502 & $3.73 \mathrm{E}-01$ & $1.77 \mathrm{E}-01$ & $6.89 \mathrm{E}-02$ & $2.67 \%$ \\
521 & $4.77 \mathrm{E}-01$ & $9.32 \mathrm{E}-02$ & $5.82 \mathrm{E}-02$ & $0.81 \%$ \\
541 & $5.41 \mathrm{E}-01$ & $4.92 \mathrm{E}-02$ & $4.76 \mathrm{E} 92$ & $0.28 \%$ \\
554 & $5.73 \mathrm{E}-01$ & $3.22 \mathrm{E}-02$ & $4.06 \mathrm{E}-02$ & $0.36 \%$ \\
601 & $6.35 \mathrm{E}-01$ & $7.24 \mathrm{E}-03$ & $1.58 \mathrm{E}-02$ & $0.61 \%$ \\
650 & $6.66 \mathrm{E}-01$ & $1.47 \mathrm{E}-03$ & $0.00 \mathrm{E}+00$ & $0.54 \%$ \\
723 & $6.89 \mathrm{E}-01$ & $1.41 \mathrm{E}-04$ & $0.00 \mathrm{E}+00$ & $0.80 \%$ \\
782 & $6.81 \mathrm{E}-01$ & $2.07 \mathrm{E}-05$ & $0.00 \mathrm{E}+00$ & $0.56 \%$ \\
816 & $6.76 \mathrm{E}-01$ & $7.15 \mathrm{E}-06$ & $0.00 \mathrm{E}+00$ & $0.23 \%$ \\
\hline
\end{tabular}


Schott Fiber Optic RWY 47 (Ce Doped)

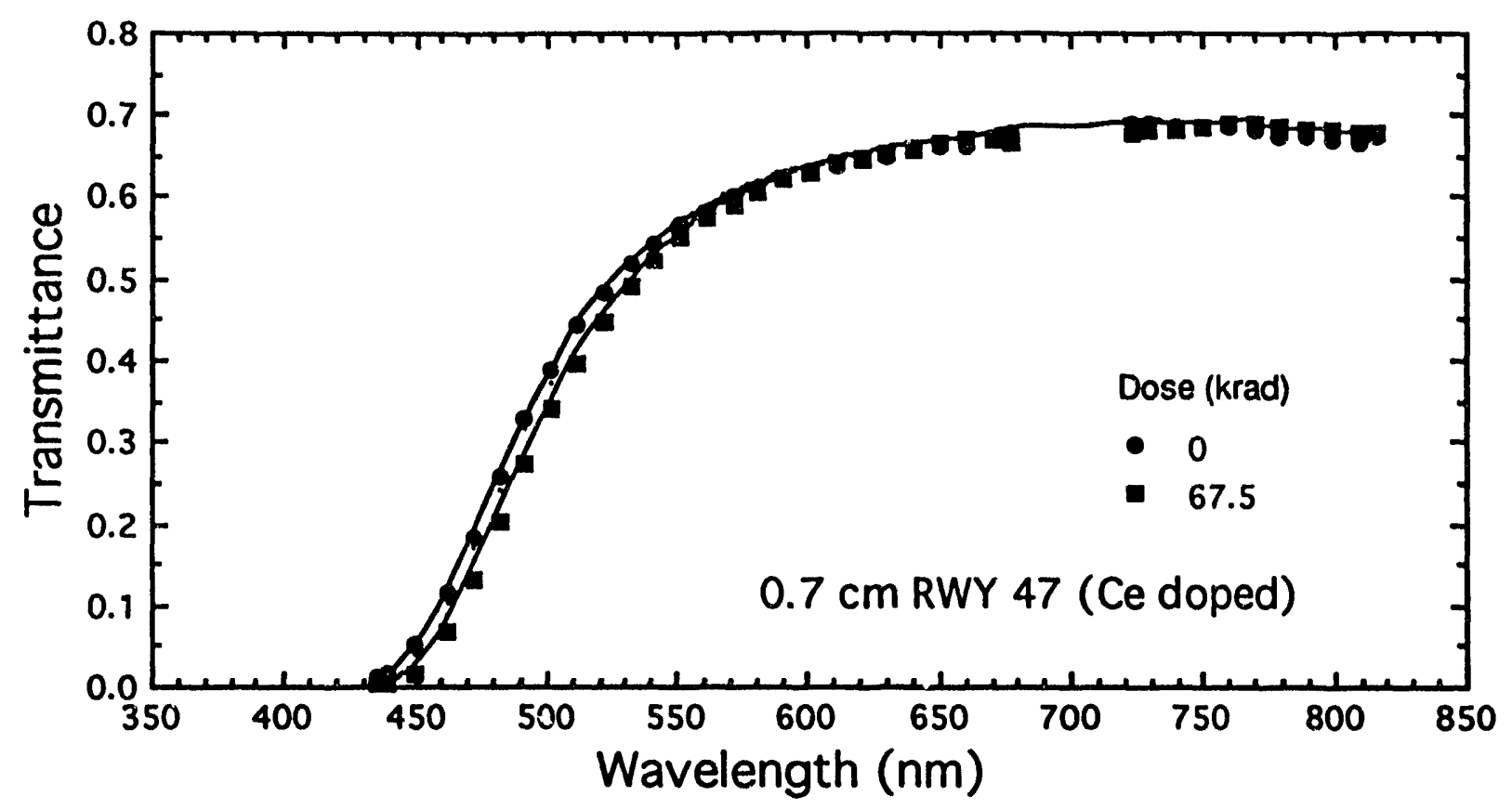

Fig. 9a: Transmittance vs. wavelength for $0.7 \mathrm{~cm}$ of Ce doped RWY 47 fiber optics (Schot Fiber Optic) after exposure to various radiation doses. The plotted points are measured data; solid lines are the fits using the "best fit" values to the radiation induced absorption parameters appearing in Eqs. 1 and 2. (The "best fit" valnes are shown as dots in the plots below.)
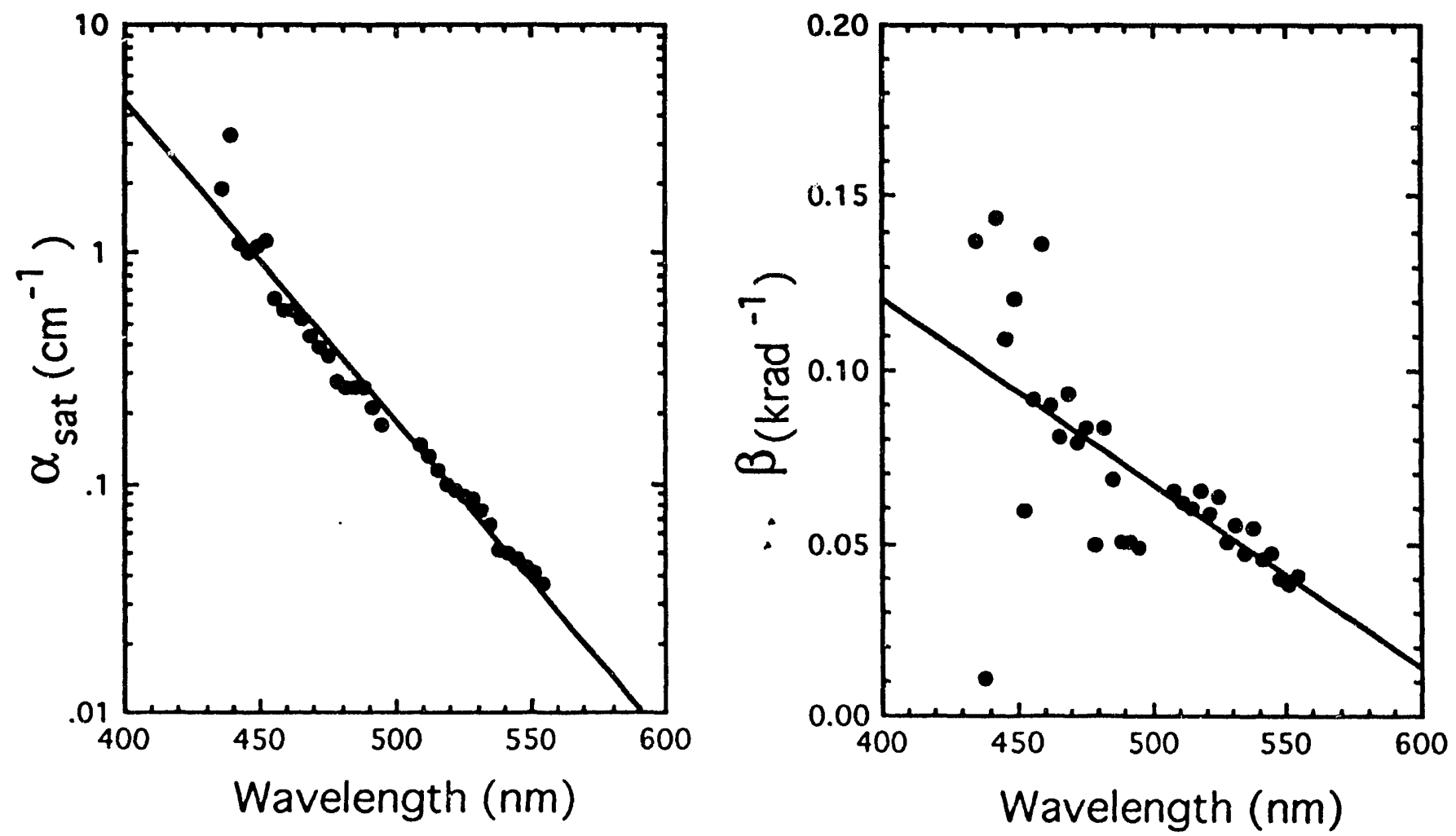

Fig. 9b: Radiation Induced Absorption Parameters vs. Wavelength for Ce Doped RWY 47. Dots are values given by least squares fits, solid lines are suggested analytic approximations to the fitted data. 


\section{Schott BK 7}

Table 10a: Least Squares Fit to $T_{0}, \alpha_{\text {sat }}$ and $\beta$, Schott BK 7

\begin{tabular}{|c|c|c|c|c|}
\hline$\lambda(\mathrm{nm})$ & $\mathrm{T}_{0}$ & $\alpha_{\text {sat }}\left(\mathrm{cm}^{-1}\right)$ & $\beta\left(\mathrm{krad}^{-1}\right)$ & error $(\mathrm{ms} \%)$ \\
\hline 380 & $9.09 \mathrm{E}-01$ & $8.23 \mathrm{E}+00$ & $2.52 \mathrm{E}-03$ & $0.00 \%$ \\
400 & $9.13 \mathrm{E}-01$ & $1.45 \mathrm{E}+01$ & $1.17 \mathrm{E}-03$ & $0.01 \%$ \\
440 & $9.15 \mathrm{E}-01$ & $9.56 \mathrm{E}+00$ & $1.41 \mathrm{E}-03$ & $0.02 \%$ \\
480 & $9.18 \mathrm{E}-01$ & $1.36 \mathrm{E}+01$ & $7.79 \mathrm{E}-04$ & $0.03 \%$ \\
520 & $9.19 \mathrm{E}-01$ & $5.41 \mathrm{E}+00$ & $1.60 \mathrm{E}-03$ & $0.03 \%$ \\
560 & $9.20 \mathrm{E}-01$ & $4.06 \mathrm{E}+00$ & $1.72 \mathrm{E}-03$ & $0.02 \%$ \\
600 & $9.21 \mathrm{E}-01$ & $3.27 \mathrm{E}+00$ & $1.73 \mathrm{E}-03$ & $0.02 \%$ \\
680 & $9.22 \mathrm{E}-01$ & $1.84 \mathrm{E}+00$ & $1.74 \mathrm{E}-03$ & $0.08 \%$ \\
760 & $9.23 \mathrm{E}-01$ & $7.31 \mathrm{E}-01$ & $1.77 \mathrm{E}-03$ & $0.12 \%$ \\
840 & $9.22 \mathrm{E}-01$ & $3.14 \mathrm{E}-01$ & $1.98 \mathrm{E}-03$ & $0.20 \%$ \\
920 & $9.22 \mathrm{E}-01$ & $1.49 \mathrm{E}-01$ & $2.98 \mathrm{E}-03$ & $0.30 \%$ \\
1000 & $9.22 \mathrm{E}-01$ & $9.36 \mathrm{E}-02$ & $3.84 \mathrm{E}-03$ & $0.33 \%$ \\
1100 & $9.22 \mathrm{E}-01$ & $6.88 \mathrm{E}-02$ & $5.46 \mathrm{E}-03$ & $0.37 \%$ \\
1200 & $9.22 \mathrm{E}-01$ & $5.92 \mathrm{E}-02$ & $6.23 \mathrm{E}-03$ & $0.37 \%$ \\
\hline
\end{tabular}

Table 10b: Analytic Approximations for $\alpha_{\text {sat }}$ and $\beta$, Schott BK 7 $\alpha_{\text {sat }}=254.82 \exp (-.0075184 \lambda) \mathrm{cm}^{-1}, \beta=.0015 \mathrm{krad}^{-1}$

\begin{tabular}{|c|c|c|c|c|}
\hline$\lambda(\mathrm{nm})$ & $\mathrm{T}_{0}$ & $\alpha_{\text {sat }}\left(\mathrm{cm}^{-1}\right)$ & $\beta\left(\mathrm{krad}^{-1}\right)$ & error $(\mathrm{ms} \%)$ \\
\hline 380 & $9.09 \mathrm{E}-01$ & $1.46 \mathrm{E}+01$ & $1.50 \mathrm{E}-03$ & $8.78 \%$ \\
400 & $9.13 \mathrm{E}-01$ & $1.26 \mathrm{E}+01$ & $1.50 \mathrm{E}-03$ & $7.36 \%$ \\
440 & $\varsigma .15 \mathrm{E}-01$ & $9.32 \mathrm{E}+00$ & $1.50 \mathrm{E}-03$ & $2.06 \%$ \\
480 & $9.18 \mathrm{E}-01$ & $6.90 \mathrm{E}+00$ & $1.50 \mathrm{E}-03$ & $2.21 \%$ \\
520 & $9.19 \mathrm{E}-01$ & $5.11 \mathrm{E}+00$ & $1.50 \mathrm{E}-03$ & $4.38 \%$ \\
560 & $9.20 \mathrm{E}-01$ & $3.78 \mathrm{E}+00$ & $1.50 \mathrm{E}-03$ & $5.62 \%$ \\
600 & $9.21 \mathrm{E}-01$ & $2.80 \mathrm{E}+00$ & $1.50 \mathrm{E}-03$ & $6.21 \%$ \\
680 & $9.22 \mathrm{E}-01$ & $1.53 \mathrm{E}+00$ & $1.50 \mathrm{E}-03$ & $3.83 \%$ \\
760 & $9.23 \mathrm{E}-01$ & $8.41 \mathrm{E}-01$ & $1.50 \mathrm{E}-03$ & $0.14 \%$ \\
840 & $9.22 \mathrm{E}-01$ & $4.61 \mathrm{E}-01$ & $1.50 \mathrm{E}-03$ & $0.41 \%$ \\
920 & $9.22 \mathrm{E}-01$ & $2.52 \mathrm{E}-01$ & $1.50 \mathrm{E}-03$ & $0.36 \%$ \\
1000 & $9.22 \mathrm{E}-01$ & $1.38 \mathrm{E}-01$ & $1.50 \mathrm{E}-03$ & $0.64 \%$ \\
1100 & $9.22 \mathrm{E}-01$ & $6.52 \mathrm{E}-02$ & $1.50 \mathrm{E}-03$ & $1.08 \%$ \\
1200 & $9.22 \mathrm{E}-01$ & $3.08 \mathrm{E}-02$ & $1.50 \mathrm{E}-03$ & $1.24 \%$ \\
\hline
\end{tabular}




\section{Schott BK 7}

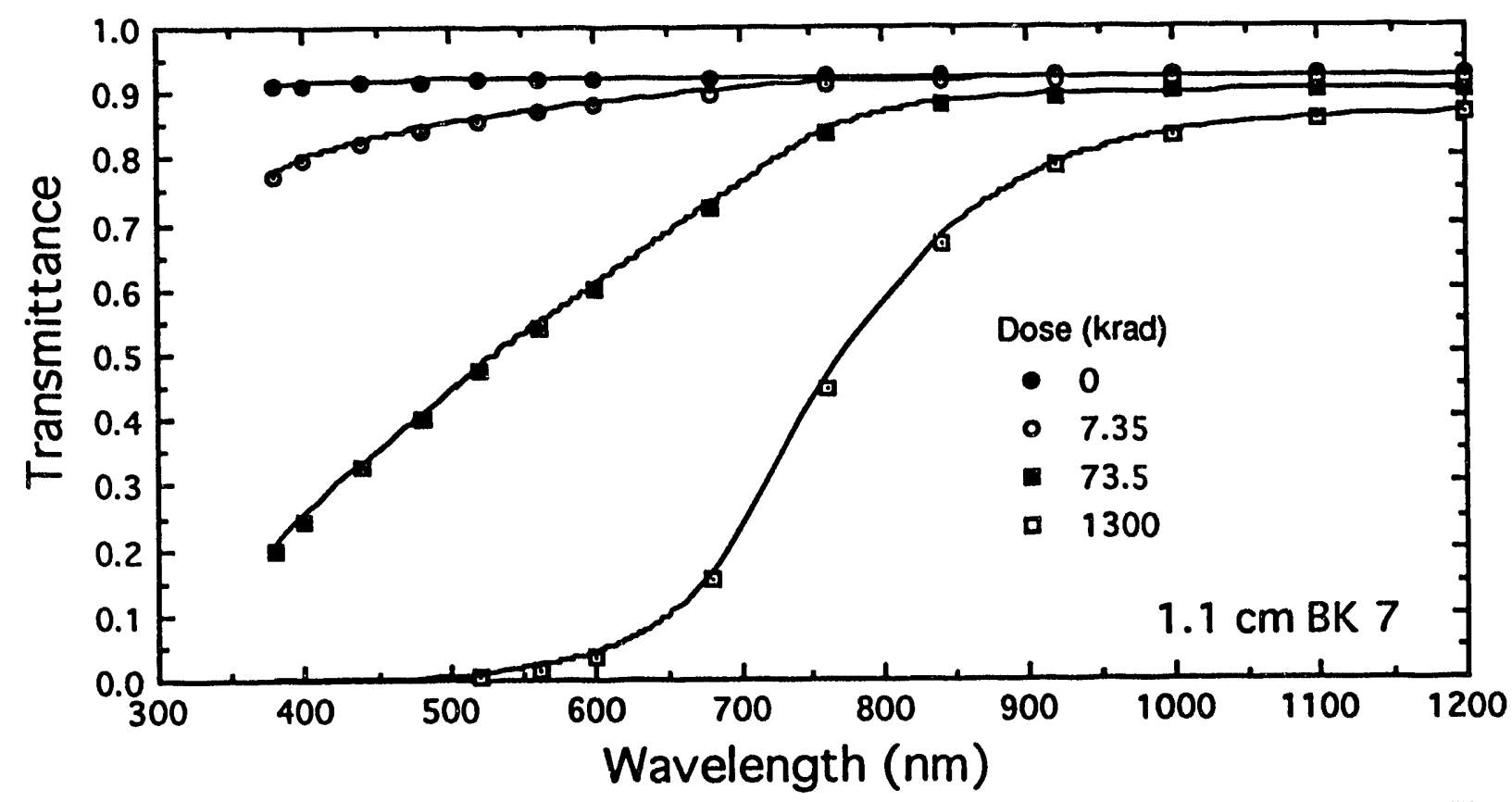

Fig. 7a: Transmittance vs. wavelength for $1.11 \mathrm{~cm}$ of Schot BK 7 after exposure to various radiation doses. The plotted points are measured data; the solid lines are the fits using the "best fit" values to the radiation induced absorption parameters appearing in Eqs. 1 and 2. (The "best fit" values are shown as dots in the plots below.)
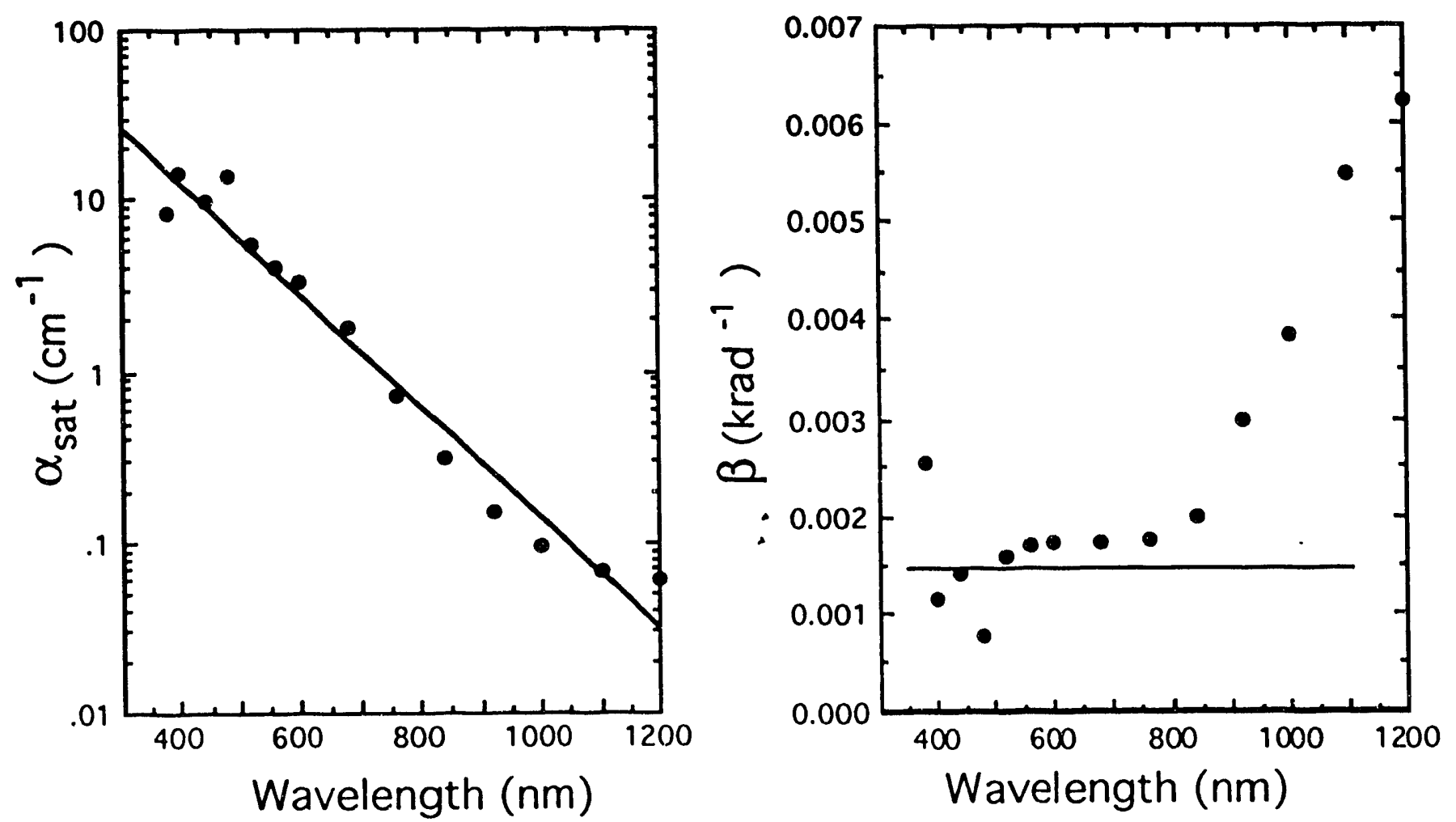

Fig. 7b: Radiation Induced Absorption Parameters vs. Wavelength for Scholt BK 7. Dots are values given by least squares fits, solid lines are suggested analytic approximations to the fitted data. 


\section{ACKNOWLEDGMENTS}

We thank Marcia Kellam, who made all the measurements upon which this analysis is based, and Calvin Gillespie for his guidance. We are especially indebted to Nicholas Colella who motivated and supported this research.

This work was performed under the auspices of the U.S. Department of Energy by the University of California, Lawrence Livermore National Laboratory under Contract W-7405

\section{REFERENCES}

Coakley 1992: Peter Coakley/JAYCOR, Private Communication

Levy 1985: P. Levy, SPIE transactions: Radiation Effects in Optical Materials Vol. 541 p 2-24 (1985)

Schott c1980: "Optical Glass, No. 3111" catalogue available from Schott Optical Glass, Duryea Pa.

Schott c1990: "Radiation Resistant Glasses" brochure available from Schott Optical Glass, Duryea Pa.

Stroud 1966: J.S. Stroud, J.W.H. Schreurs and R.F. Tucker "Charge Trapping and the Electronic Structure of Glass" Proc. of the VII International Congress on Glass (Brussels, 1965), Gordon and Breach, N.Y. (1966)

Treadaway 1975a: M.J. Treadaway, B.C. Passenheim and B.D. Kitterer "Transient Radiation Effects in Optical Materials" Intelcom Rad Tech Inc. Report No. SAMSO-TR-75-174. This is a very thorough and information packed report on a series of experiments that addressed particularly the study of the effects of radiation on optical materials of interest in connection with spacecraft.

Treadaway 1975b: M.J. Treadaway, B.C. Passenheim, and B.D. Kitterer "Luminescence and Absorption of Electron-Irradiated Common Optical Glasses, Sapphire, and Quartz" IEEE Transaction on Nuclear Science, Vol. NS-22, December 1975. This article summarizes much of the material reported in Treadaway (1975a) and is more readily available.

Wirtenson/White 1992: "Effects of Ionizing Radiation on Selected Optical Materials: An Overview", Lawrence Livermore National Laboratory Report No. UCRL-ID-111453 (July 1992)

Wirtenson/White 1993: "Calibration of the Standards and Calibration Laboratory's Co ${ }^{60}$ Ratiation Pool", Lawrence Livermore National Laboratory Report No. UCRL-ID-112675 (January 1993) 

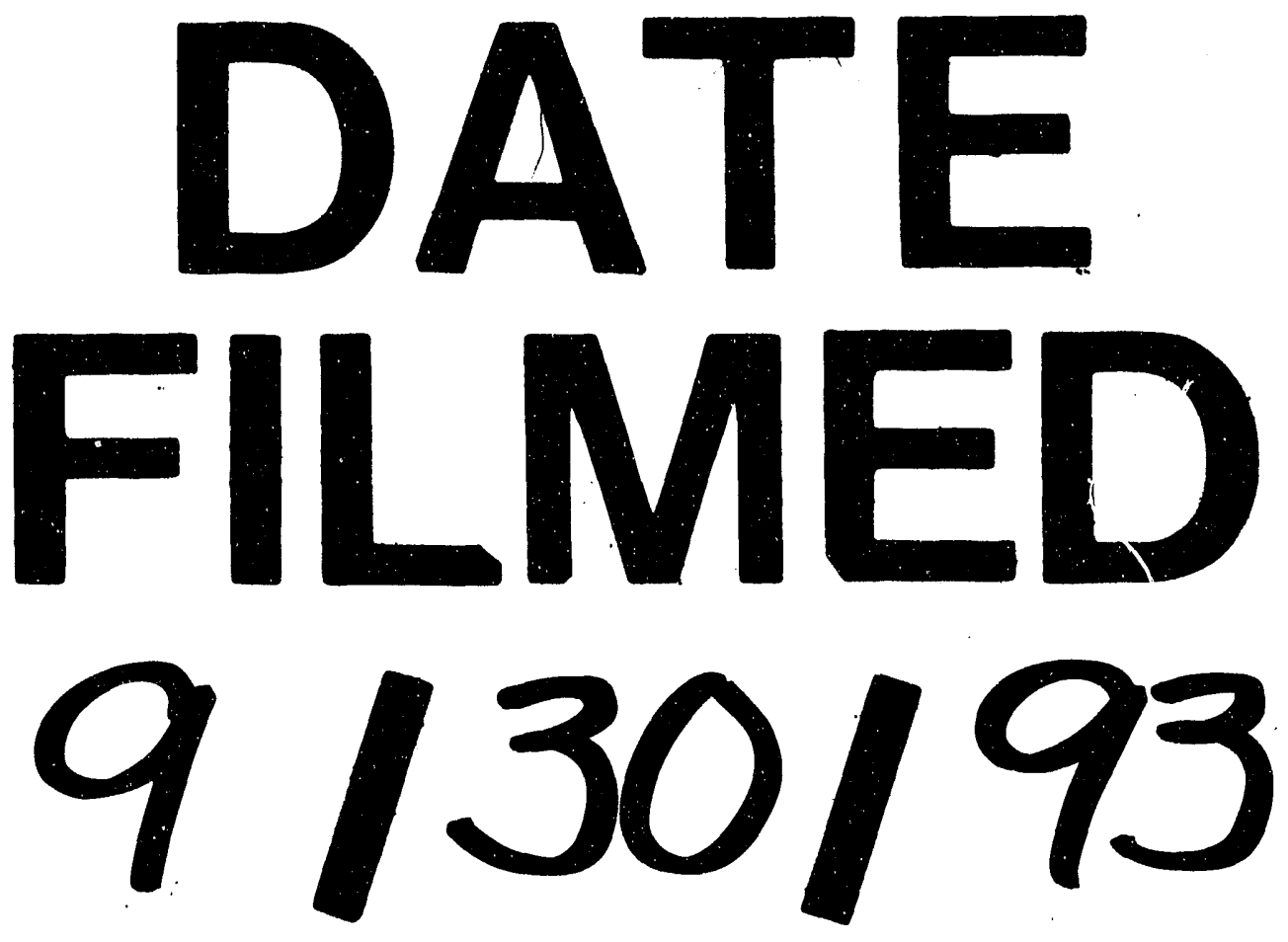
